\title{
Activation of Extracellular Signal-Regulated Kinase in the Anterior Cingulate Cortex Contributes to the Induction and Expression of Affective Pain
}

\author{
Hong Cao, ${ }^{1 \star}$ Yong-Jing Gao, ${ }^{1,2 \star}$ Wen-Hua Ren, ${ }^{1}$ Ting-Ting Li, ${ }^{1}$ Kai-Zheng Duan, ${ }^{1}$ Yi-Hui Cui, ${ }^{3}$ Xiao-Hua Cao, ${ }^{3}$ \\ Zhi-Qi Zhao, ${ }^{1}$ Ru-Rong Ji, ${ }^{2}$ and Yu-Qiu Zhang ${ }^{1}$ \\ ${ }^{1}$ Institute of Neurobiology, Institutes of Brain Science and State Key Laboratory of Medical Neurobiology, Fudan University, Shanghai 200032, China, \\ ${ }^{2}$ Department of Anesthesiology, Brigham and Women's Hospital, Harvard Medical School, Boston, Massachusetts 02115, and ${ }^{3}$ Shanghai Institute of Brain \\ Functional Genomics, Key Laboratory of Brain Functional Genomics, Ministry of Education, and Science and Technology Commission of Shanghai \\ Municipality, East China Normal University, Shanghai 200062, China
}

The anterior cingulate cortex (ACC) is implicated in the affective response to noxious stimuli. However, little is known about the molecular mechanisms involved. The present study demonstrated that extracellular signal-regulated kinase (ERK) activation in the ACC plays a crucial role in pain-related negative emotion. Intraplantar formalin injection produced a transient ERK activation in laminae V-VI and a persistent ERK activation in laminae II-III of the rostral ACC (rACC) bilaterally. Using formalin-induced conditioned place avoidance (F-CPA) in rats, which is believed to reflect the pain-related negative emotion, we found that blockade of ERK activation in the rACC with MEK inhibitors prevented the induction of F-CPA. Interestingly, this blockade did not affect formalin-induced two-phase spontaneous nociceptive responses and CPA acquisition induced by electric foot-shock or U69,593, an innocuous aversive agent. Upstream, NMDA receptor, adenylyl cyclase (AC) and phosphokinase A (PKA) activators activated ERK in rACC slices. Consistently, intra-rACC microinjection of AC or PKA inhibitors prevented F-CPA induction. Downstream, phosphorylation of cAMP response element binding protein (CREB) was induced in the rACC by formalin injection and by NMDA, AC and PKA activators in brain slices, which was suppressed by MEK inhibitors. Furthermore, ERK also contributed to the expression of pain-related negative emotion. Thus, when rats were re-exposed to the conditioning context for retrieval of pain experience, ERK and CREB were reactivated in the rACC, and inhibiting ERK activation blocked the expression of F-CPA. All together, our results demonstrate that ERK activation in the rACC is required for the induction and expression of pain-related negative affect.

\section{Introduction}

Pain is evoked by noxious body stimuli or conditioned stimuli that predict noxious stimulation (Vogt, 2005). Many forms of pain are associated with varying severities and qualities of emotion distress. Clinical observations are increasingly indicating that in chronic pain patients, pain-related negative affect is more disabling than pain itself (Crombez et al., 1999). Recently, painrelated negative affect has gained more attention.

Accumulating evidence suggests that the anterior cingulate

\footnotetext{
Received Sept. 9, 2008; revised Jan. 11, 2009; accepted Feb. 12, 2009.

This work was supported by the National Natural Science Fund of China (NSFC Grants 30425022, 30528019, 30870835, 30821002, 30670682, and 30500153), National Basic Research Program of China (Grants 2006CB500807, 2007CB512303, and 2007(B512502), Program for Changjiang Scholars and Innovative Research Team in University (PCSIRT), and National Institutes of Health Fogarty International Research Collaboration Award (FIRCA Grant TW7180). We thank Prof. B. M. Li's group for technical support in the Morris water maze and M. Han for neuropathic pain experiments.

${ }^{*}$ H.C. and Y.-J.G. contributed equally to this work.

Correspondence should be addressed to either of the following: Dr. Yu-Qiu Zhang, Institute of Neurobiology, Institutes of Brain Science, Fudan University, 138 Yi Xue Yuan Road, Shanghai 200032, China, E-mail: yuqiuzhang@fudan.edu.cn; or Ru-Rong Ji, Department of Anesthesiology, Brigham and Women's Hospital, Harvard Medical School, 75 Francis Street, Boston, MA 02115, E-mail: rrj@@zeus.bwh.harvard.edu. D0I:10.1523/JNEUROSCI.4300-08.2009

Copyright $\odot 2009$ Society for Neuroscience $\quad$ 0270-6474/09/293307-15\$15.00/0
}

cortex (ACC) is a key structure for pain affect (Price, 2000). Intrarostral ACC (rACC) injection of kynurenic acid, a wide-spectrum excitatory amino acid receptor antagonist, blocked the acquisition of formalin-induced conditioned place avoidance (F-CPA) (Johansen and Fields, 2004). Our previous studies also demonstrated that blockade of NMDA but not AMPA/KA receptors in the rACC eliminated F-CPA, suggesting that activation of NMDA receptor in the rACC is necessary for the induction of painrelated negative emotion (Lei et al., 2004a; Ren et al., 2006). NMDA receptor is highly expressed in the ACC and plays a critical role in mediating long-lasting synaptic plasticity (Monyer et al., 1994; Wei et al., 2001; Zhao et al., 2005). It is also generally believed that maintenance of long-term synaptic plasticity requires gene expression and protein synthesis (Bailey et al., 1999). To understand the mechanisms underlying NMDA-mediated affective pain, it is important to identify the signaling pathways that couple NMDA receptors to gene expression.

Extracellular signal-regulated kinase (ERK), a family member of mitogen-activated protein kinases (MAPKs), has been implicated in NMDA-dependent synaptic plasticity, such as learning/ memory and pain hypersensitivity in the hippocampus and spinal cord (Ji et al., 1999; Karim et al., 2001, Krapivinsky et al., 2003; 
Kawasaki et al., 2004; Wei et al., 2006). In cell lines and cultured neurons, activated ERK translocates from the cytosol to the nucleus where it phosphorylates cAMP response ele3ment binding protein (CREB), subsequently activating cAMP response element (CRE)-mediated gene transcription (Treisman, 1996; Impey et al., 1998; Bozon et al., 2003). A series of work have highlighted a critical role of the ERK cascades in triggering gene transcription after synaptic activation (Sweatt, 2001; Ji et al., 2002). Moreover, ERK is known to integrate other signaling pathways, such as cAMP/protein kinase A (PKA) pathway (Hu and Gereau, 2003; Kawasaki et al., 2004).

Given the similarities in molecular mechanisms underlying synaptic plasticity in different regions in the CNS (Ji et al., 2003), we hypothesized that molecular mechanism underlying painrelated negative emotion in the rACC may share common signaling pathways with nociceptive sensitization in the spinal cord and learning/memory in the hippocampus. In this study, using a rodent formalin pain model that allows simultaneous measurement of a learned behavior that directly reflects the pain affect (F-CPA) and "acute" formalin-induced "pain" behavior (paw lifting, licking, and flinching) that reflects the noxious stimulus parameters (intensity, localization) (Johansen et al., 2001), we demonstrated that the NMDA receptor-cAMP-PKA-ERK signaling pathway in the rACC is also involved in pain-related negative affect. In particular, ERK activation in the rACC is required both for the induction and expression of affective pain but not for nociceptive pain.

\section{Materials and Methods}

Animals and reagents. Experiments were performed on adult (weighing $220-250 \mathrm{~g}$ ) and young (4-week-old) male Sprague Dawley rats for in vivo studies and brain slice studies, respectively. Animals were obtained from Experimental Animal Center of Fudan University and were on a 12:12 light-dark cycle with a room temperature of $23 \pm 1^{\circ} \mathrm{C}$, and received food and water ad libitum. Before experimental manipulations, the animals were given a period of $7 \mathrm{~d}$ to adjust to the new surroundings. All experiments were performed in according with the guidelines of the International Association for the Study of Pain, and were approved by the Shanghai Animal Care and Use Committee.

All the reagents used in the present study including NMDA, APv (DL2-amino-5-phosphonovaleric acid), U69,593 (5 $\alpha, 7 \alpha, 8 \beta)-(+)-N$ methyl- $N$-(7-(1-pyrrolidinyl)-1-oxaspiro(4,5)dec-8-yl) benzeneecetamide), PD98059 (2-(2-amino-3-methoxyphenyl)-4H-1-benzopyran4-one), U0126 (1,4-diamino-2,3-dicyano-1,4-bis (o-aminophenylmercapto) butadiene ethanolate), Sp-cAMP (Sp-Adenosine 3',5'-cyclic monophosphothioate triethyammonium salt hydrate), Rp-cAMP (Rp-Adenosine 3',5'-cyclic monophosphorothiote triethyammonium salt hydrate), SQ22536 (9-(Tetrahydro-2-furanyl)-9H-purin-6-amine), and forskolin were purchased from Sigma. U69,593 $(0.16 \mathrm{mg} / \mathrm{ml})$ was dissolved in $20 \%$ propylene glycol/saline and stirred overnight. PD98059 (10 mM) and U0126 (2 mM) were dissolved in 10\% and 35\% dimethylsulfoxide (DMSO), respectively. NMDA, APv, Sp-cAMP, Rp-cAMP, forskolin, and SQ22536 were dissolved in normal saline (NS). The DMSO concentration used in slice experiment was $0.1-0.2 \%$.

Conditioned place avoidance. Conditioned place avoidance (CPA) was conducted as described previously (Gao et al., 2004), with slight modifications. The place conditioning apparatus consists of three opaque acrylic compartments. Two large ones are conditioning compartments $(30 \times 30 \times 30 \mathrm{~cm})$ and a smaller one is a neutral choice compartment $(15 \times 20 \times 30 \mathrm{~cm}$, length $\times$ width $\times$ height $)$. The conditioning compartments are placed in parallel and separated by a wall with a square door $(10 \times 10 \mathrm{~cm})$. The neutral compartment is laid in front of the two conditioning compartments with two doors $(10 \times 10 \mathrm{~cm})$ to them. A movable transparent ceiling covers each compartment. The two conditioning compartments are both painted black, but one is decorated with a transverse white band and contains an odor produced by $1.0 \%$ acetic acid; the other is decorated with a white vertical band and has an odor of cinnamon. The floors of the conditioning compartments are also different: one is made from Plexiglas, and the other is from a polyester board with a metal band on it, which can provide an electric shock. Thus, the two conditioning compartments have distinct visual, olfactory and tactile cues. The neutral compartment is white, absent of distinctive odor and has a solid acrylic floor with a slope.

The experimental process consists of three distinct sessions: a preconditioning session, a conditioning session and a test (postconditioning) session. CPA task processing takes $4 \mathrm{~d}$. Day 1 is a preconditioning day. At the beginning, a rat was placed in the neutral compartment. After habituating for $2 \mathrm{~min}$, the entrance to each conditioning compartment was opened. When the rat enters any conditioning compartment, the doors connecting neutral and conditioning compartments were closed. The rat was allowed to explore the two conditioning compartments freely for 15 $\mathrm{min}$. A timer automatically recorded the time spent in each of the compartments in a blind manner. Rats that spent $>80 \%$ (720 s) on one side on that day were eliminated from the subsequent experiments. Day 2 and 3 are conditioning days. For formalin injection and foot-shock experiments, the rat received no treatment on day 2 , and was randomly confined to one of the conditioning compartments for $45 \mathrm{~min}$. On day 3 , for producing formalin-induced CPA (F-CPA), the rat was given a unilateral hindpaw intraplantar injection of $5 \%$ formalin $(50 \mu \mathrm{l})$ or NS (control), and then restrained in the other conditioning compartment for $45 \mathrm{~min}$. For electric foot-shock-induced CPA (S-CPA), the rat received an electric shock $(0.5 \mathrm{~mA}$ for $2 \mathrm{~s})$ every $8-10 \mathrm{~min}$ in the other conditioning compartment during the 45 -min training session. For U69,593 experiments, rats received no treatment in the morning on day 2 , and were randomly confined to one of the conditioning compartments for $1 \mathrm{~h}$. After $5 \mathrm{~h}$, in the afternoon, the rats were given a subcutaneous injection of $\mathrm{U} 69,593(0.16 \mathrm{mg} / \mathrm{kg})$ and then confined in the other conditioning compartment for $1 \mathrm{~h}$. On day 3 , the same trials as day 2 were repeated. Both the treatments (formalin, shock, U69,593, vehicle, or no treatment) and the compartments were counterbalanced. Day 4 is postconditioning day. The procedure is the same as day 1 . The time animals spent in each compartment was measured.

F-CPA retrieval. Rats received the same training trials in days $1-3$ of conditioning procedure as F-CPA. But on day 4 , they were placed into the formalin-paired compartment for $10 \mathrm{~min}$ to retrieve formalin-induced pain experience. Sham CPA retrieval control rats received the same conditioning procedure as F-CPA retrieval, but on day 4, they were placed into the nonformalin-paired compartment for $10 \mathrm{~min}$. Nonretrieval rats received the same training trials as F-CPA retrieval, but on day 4, they were allowed to remain in their home cages without 10 min F-CPA retrieval before kill.

Morris water maze task. The water maze consists of a black round tank, which has a diameter of $150 \mathrm{~cm}$ and height of $54 \mathrm{~cm}$ and is filled with water $\left(24 \pm 2^{\circ} \mathrm{C}\right)$ to a depth of $38 \mathrm{~cm}$. The water is made opaque so that the submerged platform $(9.0 \mathrm{~cm}$ in diameter, $2.0 \mathrm{~cm}$ below the water surface) is invisible. The training and testing protocols were essentially as described (Jin et al., 2005). The training procedure consists of two sessions with a $30 \mathrm{~min}$ interval in between, each session consisting of six consecutive trials. The submerged platform is located at the central position of the southeast quadrant of the tank. The starting position is randomly selected, but counterbalanced among the four positions. A rat was allowed to search for the submerged platform for $60 \mathrm{~s}$. If successful in locating the platform within $60 \mathrm{~s}$, the rat was allowed to stay on the platform for $30 \mathrm{~s}$; if not, it was directed to the platform and allowed to stay there for $30 \mathrm{~s}$. Thereafter, the rat was returned to a holding cage. The next trial began after an intertrial interval of $30 \mathrm{~s}$.

A three-trial retention test was conducted $48 \mathrm{~h}$ after the training. For each rat at each trial, the submerged platform was fixed at the target quadrant and the starting point was at the position opposite to it. Each rat was giving $60 \mathrm{~s}$ to locate the submerged platform. If successful in finding the platform within $60 \mathrm{~s}$, the rat was immediately returned to a holding cage for $60 \mathrm{~s}$ before next trial began. If unsuccessful in locating the platform within $60 \mathrm{~s}$, the rat was directed to the platform and allowed to stay there for $30 \mathrm{~s}$, and then was returned to a holding cage for $30 \mathrm{~s}$ before the next trial. 
Immediately after the retention test was completed, the rat was tested in a visible platform version of the Morris water maze. The platform was raised to above the water surface and covered with white gauze to make it highly visible. Each animal was placed on the visible platform for $30 \mathrm{~s}$ before testing. The starting position for any given rat from the groups was selected randomly, but once selected it was fixed for that rat, whereas the visible platform was randomly placed among the four quadrants. The rat was allowed to locate the visible platform for $60 \mathrm{~s}$ in each trial. If successful in finding the platform, the rat was returned immediately to a holding cage; if not, the rat was removed from water and returned to a holding cage. The next trial began after an intertrial interval of $60 \mathrm{~s}$. A total of three trials were conducted for each rat.

Navigation of each animal in the water maze was monitored using a video camera, a tracking system and tracking software (San Diego Instruments). The escape latency and swimming traces were recorded for subsequent analysis.

Formalin test. To minimize an environmental influence on performance of the F-CPA training task, we developed an automatic movement recorder by modifying a previous automatic detection system (Jett and Michelson, 1996; Xie et al., 2005), which can simultaneously record the two phases of the formalin-induced nociceptive behavioral response during F-CPA training task (Gao et al., 2004; Ren et al., 2006). Briefly, after intraplantar injection of formalin, the animal was immediately placed into the conditioning compartment on the conditioning day. There was a spring balance under the floor of each conditioning compartment. Nociceptive behavior (agitation) elicited by formalin injection, such as licking, flinching, shaking, elevating, clutching and favoring the affected paw, induces vibration of the spring balance, which can be converted into electrical signals via an electromagnetic transducer. The electrical signals were amplified, filtered $(1.0 \mathrm{kHz})$ and fed into a computer system that allowed quantitative recording of the number of agitation events and construction of time histograms. The histograms were recorded automatically for $45 \mathrm{~min}$, and the time course of changes of movement events per 5 min were plotted.

To validate the accuracy of this automatic recording, we also performed classic manual observation simultaneously. A mirror was positioned below a chamber at a $45^{\circ} \mathrm{C}$ angle for unobstructed observation of the rat's paws. The responses to formalin injection were monitored by measuring the time the animal spent on lifting, licking, and shaking the affected paw per 5 min during a 45 min observation period. A weighted pain score for each animal was calculated using the following formula (Tanimoto et al., 2003): formalin pain score $=[$ seconds spent on paw elevation $+2 \times$ (seconds spent licking or biting injected paw)]/300.

Chronic constriction injury of the sciatic nerve and measurement of thermal hyperalgesia and mechanical allodynia. Rats were deeply anesthetized with chloral hydrate $(40 \mathrm{mg} / \mathrm{kg})$. The skin of right hind limb was sterilized with iodine tincture, then $75 \%$ alcohol, and the right sciatic nerve exposed at the mid-thigh level by blunt dissection of the biceps femoris. For producing chronic constriction injury (CCI), four chromic gut $(4-0)$ ligatures were tied loosely around the nerve $\sim 1 \mathrm{~mm}$ apart, proximal to its trifurcation, as described by Bennett and Xie (1988). For sham surgery, the sciatic nerve was isolated but not ligated. After CCI or sham surgery, the overlying muscles and skin were closed respectively in layers with 4-0 silk sutures and dusted with antibiotic power.

Thermal hyperalgesia was assessed by measuring the paw withdrawal latency (PWL) in response to a radiant heat source using Hargreaves' test. Briefly, rats were placed individually into Plexiglas chambers on an elevated glass platform, under which a radiant heat source (model 336 combination unit, IITC/Life Science Instruments) was applied to the glabrous surface of the paw through the glass plate. The heat source was automatically turned off when the rat lifted the foot, allowing the measurement of paw withdrawal latency (PWL). The heat was maintained at a constant intensity, which produced a stable PWL of $\sim 10-12 \mathrm{~s}$ in normal animals. A $20 \mathrm{~s}$ cutoff was used to prevent tissue damage in the absence of a response. After acclimation to the test chambers, both hindpaws were tested independently with $10 \mathrm{~min}$ intervals between trials.

Mechanical allodynia was assessed by measuring the paw withdrawal threshold (PWT) in response to a calibrated series of von-Frey hairs (Stoelting) ranging from 0.6 to $26 \mathrm{~g}$. Animals were placed individually into wire mesh-bottom cages, and allowed to acclimatize for $\sim 30 \mathrm{~min}$. A series of 10 calibrated von Frey hairs were applied to the central region of the plantar surface of one hindpaw in ascending order $(0.6,1,1.4,2,4,6$, $8,10,15$, and $26 \mathrm{~g}$ ). A particular hair was applied until buckling of the hair occurred. This was maintained for $\sim 2 \mathrm{~s}$. The hair was applied only when the rat was stationary and standing on all four paws. A withdrawal response was considered valid only if the hindpaw was completely removed from the customized platform. Each hair was applied 5 times at $5 \mathrm{~s}$ intervals. If withdrawal responses did not occur more than twice during five applications of a particular hair, the next ascending hair in the series was applied in a similar manner. Once the hindpaw was withdrawal from a particular hair three out of the five consecutive applications, the rat was considered responsive to that hair. The PWT was defined as the lowest hair force in grams that produced at least three withdrawal responses in five tests. After the threshold was determined for one hindpaw, the same testing procedure was repeated on the other hindpaw at 5-min interval.

Intra-rACC drug infusions. Rats were anesthetized with intraperitoneal chloral hydrate $(40 \mathrm{mg} / \mathrm{kg})$, and securely placed into a sterotaxic device with bregma and lambda at a horizontal level. A 30 gauge stainless steel cannula with a 33 gauge stainless steel stylet plug was bilaterally implanted $0.5 \mathrm{~mm}$ above the rACC injection site [anteroposterior (AP) + 2.6 from bregma, mediolateral $(\mathrm{ML}) \pm 0.6$, dorsoventral $(\mathrm{DV})-2.5$ ] according to the atlas of Paxinos and Watson (1998). In some animals, double cannulae were implanted and two injections (one injection per cannula site) were applied for each side of the rACC (four injections in total) with the following coordinates: AP +3.0 from bregma, $\mathrm{ML} \pm 0.6$, $\mathrm{DV}-2.5$, and $\mathrm{AP}+1.6$ from bregma, $\mathrm{ML} \pm 0.6$, $\mathrm{DV}-2.2$, respectively. The cannula was anchored to the cranium with three stainless steel screws and dental acrylic. Animals were allowed to recover for 5-6 d, and began to gain weight before next experimental procedure. At the end of the experiment, brains were sectioned for cresyl violet staining to verify cannula position and injection site.

Microinjection was performed through a 33 gauge stainless-steel injection cannula that extended $0.5 \mathrm{~mm}$ beyond the tip of the guide cannula. The injection cannula was connected to a $1 \mu \mathrm{l}$ Hamilton syringe with PE-10 tubing. A total volume of $0.5 \mu$ l per hemisphere of either vehicle or drug was injected over a $5 \mathrm{~min}$ period. The injection cannula was left in place for an additional 5 min to minimize spread of the drug along the injection track.

To examine the effect of drugs on the induction (acquisition) of F-CPA, S-CPA, or U69,593-induced CPA, intra-rACC microinjection of drugs or vehicle was performed $30 \mathrm{~min}$ before intraplantar formalin (or foot-shock, s.c. U69,593) on conditioning day. To examine the effects of drugs on F-CPA expression, the drugs or vehicle were administered into the bilateral rACC $30 \mathrm{~min}$ or $6 \mathrm{~h}$ before F-CPA test on postconditioning day. To examine whether drugs per se produced place preference or avoidance in rats without formalin treatment, drugs or vehicle were injected into the bilateral rACC 30 min before conditioning on conditioning day. Training procedures were performed as F-CPA (described above) except that formalin was replaced by NS. To examine the effects of drugs on Morris water maze task, the drug or vehicle was administered into the bilateral rACC $30 \mathrm{~min}$ before training in water maze. To examine the effects of drugs on formalin-induced acute spontaneous nociceptive response, the drug or vehicle was administered into the bilateral rACC 30 min before intraplantar injection of formalin. To examine the effects of drugs on CCI-induced behavioral hypersensitivity, drug or vehicle was administered on day 3 after CCI operation when the thermal hyperalgesia and mechanical allodynia reliably developed, and behavioral testing was performed at $1,2,3,4$, and $5 \mathrm{~h}$ after intra-rACC injection. This behavioral testing protocol was based on our previous study showing that PD98059 produces the best anti-neuropathic pain effect at $3 \mathrm{~h}$ after injection (Zhuang et al., 2005). All behavioral tests were performed blind with respect to drugs injected.

Western blots. After defined survival times, rats were killed by overdose of Chloral hydrate $(80 \mathrm{mg} / \mathrm{kg})$ and the brain was quickly removed. The rACC was dissected on ice using a Rat Brain Matrix (Stoelting Company). Briefly, three coronal brain slices ( $1 \mathrm{~mm}$ thick) containing rACC (AP 3.7-0.7 from bregma) were cut. Then the brain slices were separated into left and right half from the sagittal suture. Finally, the rACC tissues 
were dissected using a surgical blade according to the atlas of Paxinos and Waston, and rapidly frozen in liquid nitrogen. Frozen samples were homogenized in a lysis buffer $(12.5 \mu \mathrm{l} / \mathrm{mg}$ tissue $)$ containing a mixture of protease inhibitors (Roche) and PMSF (Sigma). After incubating in ice for $30 \mathrm{~min}$, samples were centrifuged at $10,000 \mathrm{rpm}$ for $15 \mathrm{~min}$ at $4^{\circ} \mathrm{C}$. The supernatants were used for Western blotting.

Equal amount of protein $(\sim 20 \mu \mathrm{g})$ was loaded and separated in $10 \%$ Tris-Tricine SDS-PAGE gel. The resolved proteins were transferred onto polyvinilidene difluoride (PVDF) membranes (Amersham Bioscience). The membranes were blocked in $10 \%$ non-fat milk for $2 \mathrm{~h}$ at room temperature (RT), and incubated overnight at $4^{\circ} \mathrm{C}$ with mouse antiphospho-ERK (pERK1 and pERK2; 1:3000, Sigma), mouse anti-ERK (total ERK1 and ERK2; 1:100,000, Sigma), rabbit anti-pCREB (1:3000, Upstate Biotechnology), rabbit anti-CREB (total CREB; 1:1000, Sigma), or rabbit anti-pPKA RII (1:1000, Upstate Biotechnology) primary antibody. The blots were then incubated with the secondary antibody, goat anti-mouse or goat anti-rabbit IgG conjugated with horseradish peroxidase (HRP) (1:1000, Pierce), for $2 \mathrm{~h}$ at $4^{\circ} \mathrm{C}$. Signals were finally visualized using enhanced chemiluminescence (ECL, Pierce) and the blots were exposed onto X-films for 1-10 min. All Western blot analysis was performed at least three times, and consistent results were obtained.

Immunohistochemistry. After defined survival times, rats were killed by overdose of chloral hydrate $(80 \mathrm{mg} / \mathrm{kg})$ and perfused transcardially with NS followed by $4 \%$ paraformaldehyde in $0.1 \mathrm{~m}$ phosphate buffer $(\mathrm{PB}, \mathrm{pH}$ 7.4). Brains were then removed, postfixed in the same fixative for $6 \mathrm{~h}$ at $4^{\circ} \mathrm{C}$, and immersed in $10-30 \%$ gradient sucrose in $\mathrm{PB}$ for $24-48 \mathrm{~h}$ at $4^{\circ} \mathrm{C}$ for cryoprotection. Coronal sections $(30 \mu \mathrm{m})$ were cut in a cryostat (Leica 1900) and processed for immunofluorescence. Briefly, the sections were blocked with $10 \%$ donkey serum in $0.01 \mathrm{~m} \mathrm{~PB}$ saline (PBS, pH 7.4) with $0.3 \%$ Triton $\mathrm{X}-100$ for $1 \mathrm{~h}$ at $\mathrm{RT}$ and incubated overnight at $4^{\circ} \mathrm{C}$ with mouse anti-pERK (1:2000; Sigma) or rabbit anti-pCREB (1:3000; Upstate Biotechnology) primary antibody in PBS with $1 \%$ normal donkey serum and $0.3 \%$ Triton X-100. After three 15 min rinses in PBS, the sections were incubated in fluorescein isothiocyanate (FITC)-conjugated donkey anti-mouse IgG (1:200; Jackson ImmunoResearch) or rhodamine-conjugated donkey anti-rabbit IgG (1:200, Jackson ImmunoResearch) for $2 \mathrm{~h}$ at $4^{\circ} \mathrm{C}$, then washed in PBS. For pERK/NeuN, pERK/ MAP-2, pERK/OX-42, pERK/GFAP, pCREB/NeuN, pERK/pCREB, pERK/NR1, or pCREB/Fos double immunofluorescence, the sections were incubated with a mixture of rabbit anti-pERK (1:200, Cell Signaling) and mouse anti-NeuN (neuronal marker, 1:5000, Millipore), mouse anti-MAP-2 (pyramidal cell marker, 1:1000, Millipore), mouse antiOX-42 (microglia marker, 1:5000, Serotec), or mouse anti-GFAP (astrocyte marker, 1:3000, Sigma), or mouse anti-pERK (1:2000) and rabbit anti-pCREB (1:3000), or rabbit anti-pCREB (1:3000) and mouse antiNeuN (1:5000), or mouse anti-pERK (1:2000) and rabbit anti-NR1 (1: 200 , Millipore), or rabbit anti-pCREB (1:3000) and goat anti-Fos (1:200, Santa Cruz Biotechnology) overnight at $4^{\circ} \mathrm{C}$. All sections were coverslipped with a mixture of $50 \%$ glycerin in PBS, and then observed with a Leica SP2 confocal laser-scanning microscope. The specificity of immunostaining was verified by omitting the primary antibodies, and immunostaining signal disappeared after omitting primary antibodies. The specificity of primary antibodies was verified by the preabsorption experiment. Brain sections were first incubated with a mixture of pERK or pCREB primary antibody and the corresponding blocking peptide for phospho-p44/42 (10 $\mu \mathrm{g} / \mathrm{ml}$; Cell signaling) or phospho-CREB ( $20 \mu \mathrm{g} / \mathrm{ml}$; Cell Signaling) overnight, followed by secondary antibody incubation. pERK and pCREB immunostaining signal was abolished after absorption (supplemental Fig. 1, available at www.jneurosci.org as supplemental material).

Brain slice preparation. Coronal brain slices containing the rACC were obtained from young rats (4-week old). After anesthetizing with isoflurane, rats were decapitated. The brain was quickly removed and submerged in preoxygenated $\left(95 \% \mathrm{O}_{2}\right.$ and $\left.5 \% \mathrm{CO}_{2}\right)$ cold artificial CSF (ACSF) containing (mM): $126 \mathrm{NaCl}, 4.0 \mathrm{KCl}, 1.25 \mathrm{MgCl}_{2}, 26 \mathrm{NaHCO}_{3}$, $1.25 \mathrm{NaH}_{2} \mathrm{PO}_{4}, 2.5 \mathrm{CaCl}_{2}$, and 10 glucose. The osmolarity was adjusted to $300 \mathrm{mosmol} / \mathrm{L}$ and the $\mathrm{pH}$ to 7.35 . Slices ( $380 \mu \mathrm{m}$ thick) were cut with a vibratome (Leica VT1000S) and transferred to an oxygenated chamber at $\mathrm{RT}\left(22 \pm 1^{\circ} \mathrm{C}\right)$ for at least $1 \mathrm{~h}$ before further processing.

For immunohistochemical experiments, the rACC slices were treated with different agonist (or activators) at RT: $\operatorname{NMDA}(20,50$, and $100 \mu \mathrm{M})$, forskolin $(50 \mu \mathrm{M})$, or Sp-cAMP $(50 \mu \mathrm{M})$ for $10 \mathrm{~min}$. NMDA receptors antagonist $\mathrm{APv}(50 \mu \mathrm{M})$, adenylyl cyclase (AC) inhibitor SQ22536 (50 $\mu \mathrm{M})$, PKA inhibitor Rp-cAMP (50 $\mu \mathrm{M})$, or MAP kinase kinase (MEK) inhibitor PD $98059(50 \mu \mathrm{M})$ was added to ACSF 40 min before and during stimulation. Untreated or 0.2\% DMSO-treated slices served as controls. Subsequently, the slices were rapidly immersed in cold $4 \%$ paraformaldehyde and fixed for $60 \mathrm{~min}$, and then processed for immunostaining.

Quantification and statistics. For the quantification of Western signals, $\mathrm{x}$-ray films with blotting bands were scanned. Bio-Rad Image Analysis System (quantity one, Bio-Rad) was then used to measure the integrated optic density of the bands. For the quantification of immunoreactive signals, six non-adjacent sections $(30 \mu \mathrm{m})$ through the rACC were randomly selected. The numbers of pERK- and pCREB-labeled cells were counted in laminae II-VI of the rACC that was captured inside the optic field using a computerized image analysis system (Leica Qwin 500). The areas sampled within the rACC were: $520 \times 480 \mu \mathrm{m}$ in the laminae II-III and $480 \times 520 \mu \mathrm{m}$ in the laminae V-VI. Four to five rats were included in each group for quantification of Western blot and immunohistochemistry results. Differences between groups were compared using one-way ANOVA followed by post hoc Dunnett's test or using Student's $t$ test if only two groups were applied.

In the case of CPA experiments, the CPA scores represent the time spent in the treatment-paired compartment on the preconditioning day (day 1) minus the time spent in the same conditioning compartment on the postconditioning day (day 4). The differences in CPA scores among drug-treated groups were compared using Student's $t$ test for a twogroup comparison or one-way ANOVA followed by post hoc Dunnett's test for multiple group comparison. In addition, the absolute time spent in the treatment-paired compartment on the preconditioning day versus the postconditioning day was compared in training (F-CPA, S-CPA), sham training, vehicle or drug-treated animals using paired $t$ test. For formalin-induced nociceptive behaviors, the agitation-induced vibration events (using automatic movement recorder) and formalin pain scores (using manual detection) per 5 min were analyzed using a two-way (drug treatment $\times$ time) ANOVA with a post hoc comparison for analysis of the differences at each 5-min period. For CCI-induced behavioral hypersensitivity, Pre-CCI baseline measures were analyzed by one-way ANOVA. Pre-and postdrug time course measures for both hyperalgesia and allodynia were analyzed by two-way (treatment $\times$ time) ANOVA followed by a post hoc test. For Morris water maze experiments, the differences in the escape latencies to find both the submerged and visible platform between drug- and vehicle-treated groups were compared using Student's $t$ test. All data were expressed as mean \pm SEM, and the accepted level of statistical significance for all experiments was $p<0.05$.

All the behavioral testing and quantification of Western blot and immunohistochemical experiments were performed blind with respect to treatments.

\section{Results}

Intraplantar formalin injection activates ERK in the rACC

Phosphorylation of ERK has been extensively used as an indicator of ERK activation (English and Sweatt, 1996; Ji et al., 1999; Karim et al., 2001). Intraplantar injection of formalin elicited characteristic biphasic nociceptive agitation behavior including lifting, licking, shaking and biting: the first phase lasted for $\sim 5 \mathrm{~min}$ and the second phase lasted for $\sim 40 \mathrm{~min}$. The formalin-induced twophase responses were also robust using our automatic detecting system. Formalin injection also induced a rapid, persistent, and bilateral activation of ERK in the rACC. A significant increase in the number of pERK-positive cells on both sides of the rACC occurred at $3 \mathrm{~min}$, peaked at $10-30 \mathrm{~min}$, declined at $2 \mathrm{~h}$, but still remained higher than baseline after $24 \mathrm{~h}$ (Fig. $1 A, B$ ).

Interestingly, patterns of ERK activation in the different laminae of the rACC changed over time after the formalin injection. In the nonstimulated conditions, a few pERK-positive neurons were found in laminae II-III. In the early time points after forma- 
A

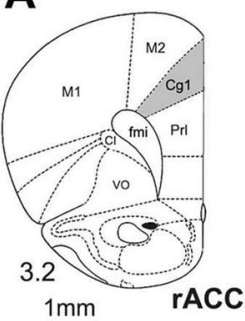

$\underline{1 \mathrm{~mm}}$
pERK

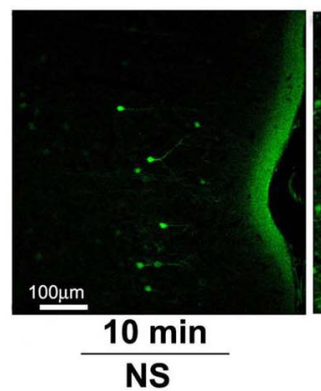

B

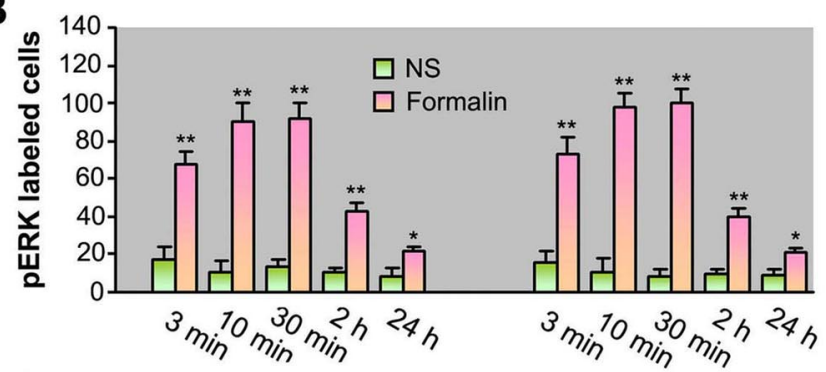

D

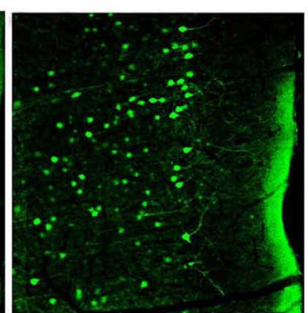

$10 \mathrm{~min}$
Contralateral

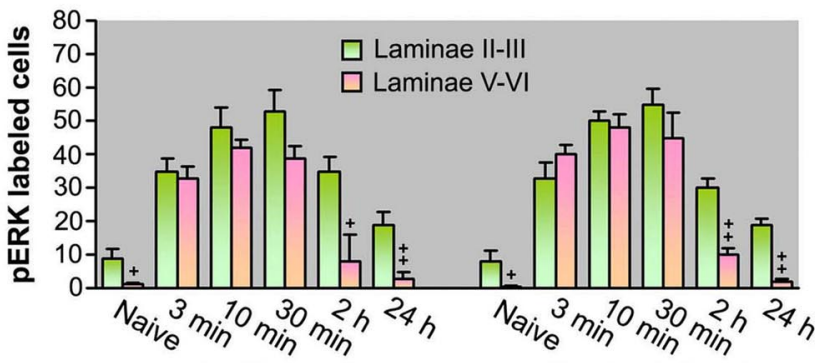

Ipsilateral

F

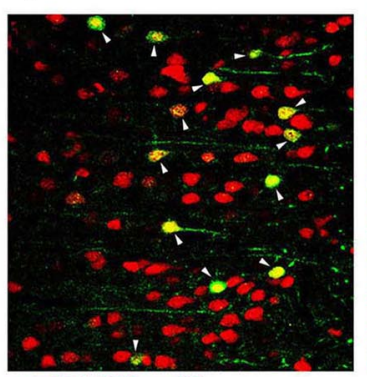

pERK/NeuN

Contralateral

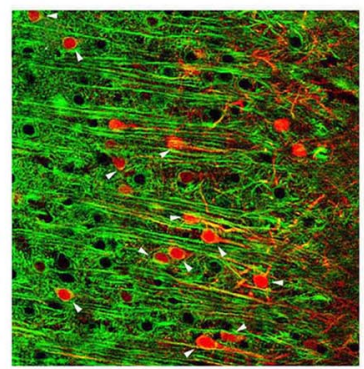

MAP-2/pERK

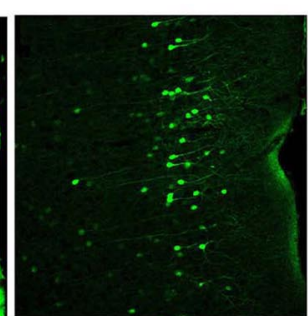

$2 \mathrm{~h}$

Formalin
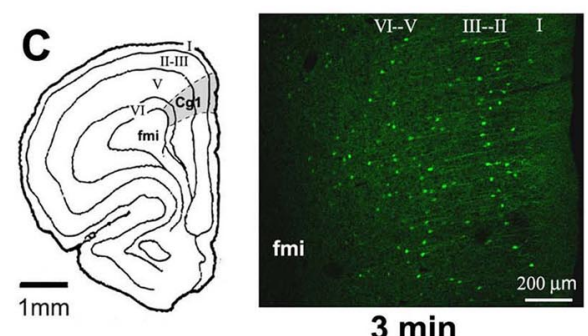

Formalin

E
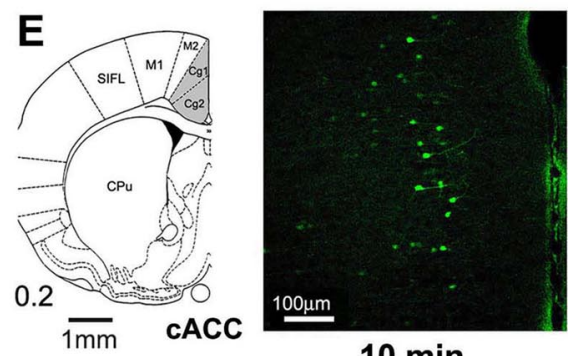

10 min

Formalin

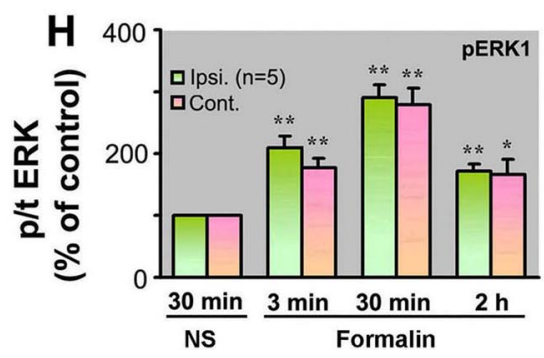

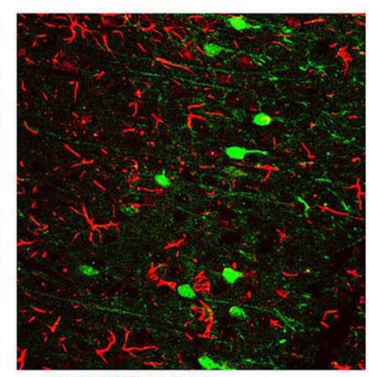

pERK/GFAP

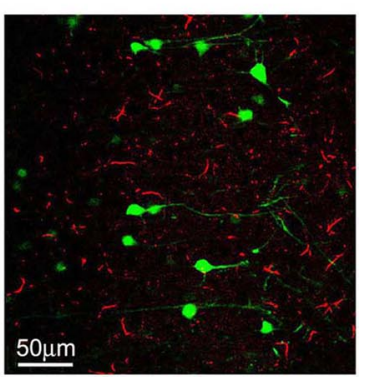

pERK/OX-42
G

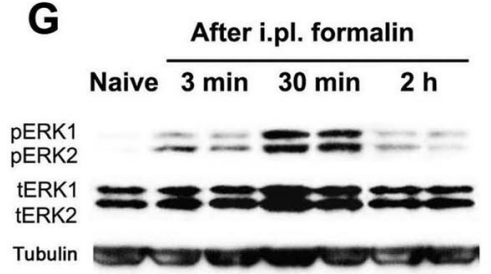

Ipsi. Cont. Ipsi. Cont. Ipsi. Cont

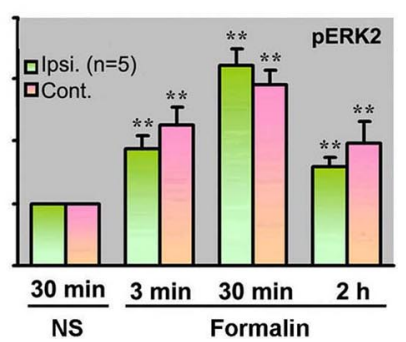

Figure 1. Time course of ERK activation in the rACC after formalin injection into a hindpaw. $A$, Immunohistochemistry for pERK in the contralateral rACC from coronal brain sections representative of NS injection at $10 \mathrm{~min}$ and formalin injection at $10 \mathrm{~min}, 2 \mathrm{~h}$, and $24 \mathrm{~h}$. Right, a high magnification inset showing pERK immunoreactivity in the nucleus (arrowhead), cytoplasm (large arrow) and dendrites (small arrow). $\boldsymbol{B}$, Numbers of pERK-positive cells in both sides of rACC at all time points after formalin injection are significantly higher than that of NS controls. ${ }^{*} p<0.05$ and ${ }^{* *} p<0.01$ compared with respective NS control $(n=5)$. C, Left, schematic drawing indicating different laminae of the rACC. Right, a low-magnification section showing $p$ ERK immunoreactivity in laminae II-VI of the rACC at 3 min after formalin injection. $\boldsymbol{D}$, Histograms showing the distribution of pERK-positive cells in all layers of the bilateral rACC. ${ }^{+} p<0.05,{ }^{++} p<0.01$ compared with the number of pERK-positive cells in laminae II-III. $\boldsymbol{E}$, pERK expression in the caudal ACC (CACC) 10 min after formalin injection. $\boldsymbol{F}$, Double immunofluorescence reveals that pERK (Figure legend continues.) 
lin injection $(3,10$, and $30 \mathrm{~min})$, pERK was increased not only in the laminae II-III, but also in the deep laminae $(\mathrm{V}-\mathrm{VI})$ of the rACC. At these time points, there was no difference between the number of pERKpositive cells in the laminae II-III and V-VI (Fig. $1 C, D$ ). In the late time points after formalin injection ( 2 and $24 \mathrm{~h}$ ), pERK increase still remained in the laminae II-III, but gradually declined in the laminae $\mathrm{V}$-VI (Fig. 1D).

Notably, we did not observe any difference in pERK levels between two sides of the rACC after formalin injection. However, only a mild increase in pERK expression was found in the caudal ACC (cACC), and pERK-positive neurons were primarily distributed in laminae II-III of the cACC (Fig. 1E). Intraplantar injection of normal saline did not induce significant ERK activation in the rACC at all the time points examined (Fig. 1 $A, B$ ).

Formalin-induced pERK was found in neurons in the rACC, because pERK was colocalized with the neuronal marker NeuN and the pyramidal cell marker MAP-2 (Fig. $1 F$ ). The pERK was found not only in the nucleus but also in cytoplasm and dendrites of neurons (Fig. $1 A$ ). We found no evidence that pERK colocalized with GFAP (glial fibrillary acidic protein), an astrocytic marker, or with $\mathrm{OX}-42$, a microglial marker (Fig. $1 F$ ).

ERK activation by formalin was confirmed by Western blot analysis. Both pERK1 (44 kDa) and pERK2 (42 kDa) significantly increased in the bilateral rACC at $3 \mathrm{~min}, 30 \mathrm{~min}$, and $2 \mathrm{~h}$ after the formalin injection (Fig. 1G,H). No significant changes in the levels of total ERK were observed for either the ERK1 or the ERK2 isoform at all the time points examined.

To determine whether contextual conditioning exposure produces ERK activation, we performed additional experiments to compare pERK levels in the rACC after intraplantar formalin with or without contextual conditioning exposure. We found that pERK levels in the rACC in these two conditions were indistinguishable (data not shown).
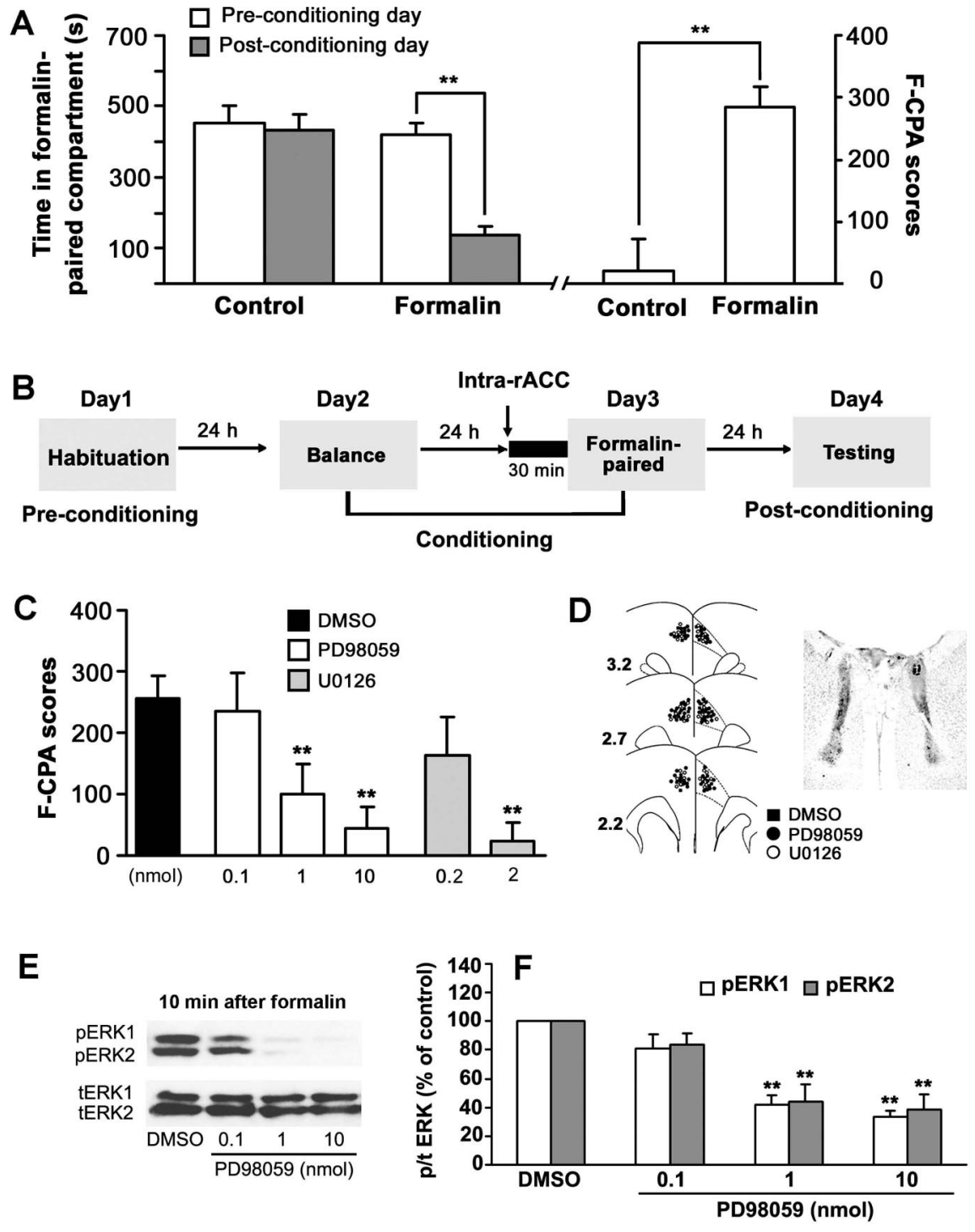

Figure 2. ERK activation in the rACC contributes to the induction of F-CPA. $\boldsymbol{A}$, Histograms showing formalin-induced CPA, as indicated by time spent in treatment (intraplantar NS or formalin)-paired compartment on preconditioning and postconditioning days (left) and CPA scores (indicated by the difference of time (seconds) spent on the preconditioning day and time spent on the postconditioning day in the treatment-paired compartment) (right). ${ }^{* *} p<0.01 . n=10$. $\boldsymbol{B}$, Schematic of the protocol for behavioral testing. $\boldsymbol{C}$, Intra-rACC microinjection of MEK inhibitors PD98059 (0.1, 1, and $10 \mathrm{nmol})$ and U0126 (0.2, and $2 \mathrm{nmol})$ before conditioning dose-dependently reduces F-CPA scores. ${ }^{* *} p<0.01$ compared with vehicle control $(n=8-12)$. D, Coronal brain section showing reconstruction of microinjection sites and cannula placement in the bilateral $r A C C$. $\boldsymbol{E}, \boldsymbol{F}$, Formalin-induced increases in levels of pERK1 and pERK2 are blocked by intra-rACC preadministration of PD98059 (1 and $10 \mathrm{nmol}$ ). Both ipsilateral and contralateral sides of the rACC were pooled together in this experiment. ${ }^{* *} p<0.01$ compared with vehicle (10\% DMSO) control $(n=4-5)$.
(Figure legend continued.) colocalizes with NeuN, and MAP-2, but does not colocalize with GFAP and OX-42 in the rACC. Arrowheads indicate double-labeled cells. G, Western blot for pERK1/2 and total ERK1/2 from the ipsilateral and contralateral side of $\mathrm{rACC}$ after formalin injection. Tubulin serves as loading control. $\boldsymbol{H}$, Densitometry analysis showing a similar increase in the levels of both $p E R K 1$ and $p E R K 2$ in the rACC after formalin injection. ${ }^{*} p<0.05$ and ${ }^{* *} p<0.01$ compared with NS control $(n=5)$. Cg1, Cingulate cortex, area 1; Cg2, cingulate cortex, area 2; $\mathrm{CPu}$, caudate-putamen; $\mathrm{M} 1$, primary motor cortex; $\mathrm{M} 2$, secondary motor cortex; Prl, prelimbic cortex; SIFL, primary somatosensory cortex, forelimb region; V0, ventral orbital cortex. Values are expressed as mean \pm SEM.

\section{ERK activation in the rACC is required for the acquisition} of F-CPA

When formalin injection was paired with a particular compartment in the place conditioning apparatus, rats spent less time in this compartment on the postconditioning day compared with the preconditioning day $(388.7 \pm 37.03 \mathrm{~s}$ preconditioning vs $110.5 \pm 30.94$ s postconditioning, Paired $t$ test, $p<0.01, n=8$ ). Control animals with intraplantar injection of NS did not produce CPA (Fig. 2A). The differences in the CPA scores [the time spent in the treatment-paired compartment on the preconditioning day (day 1) minus the time spent in the same conditioning compartment on the postconditioning day (day 4)] between formalin- and NS-treated groups were statistically significant (Student's $t$ test, $p<0.05, n=6-8$ ). 

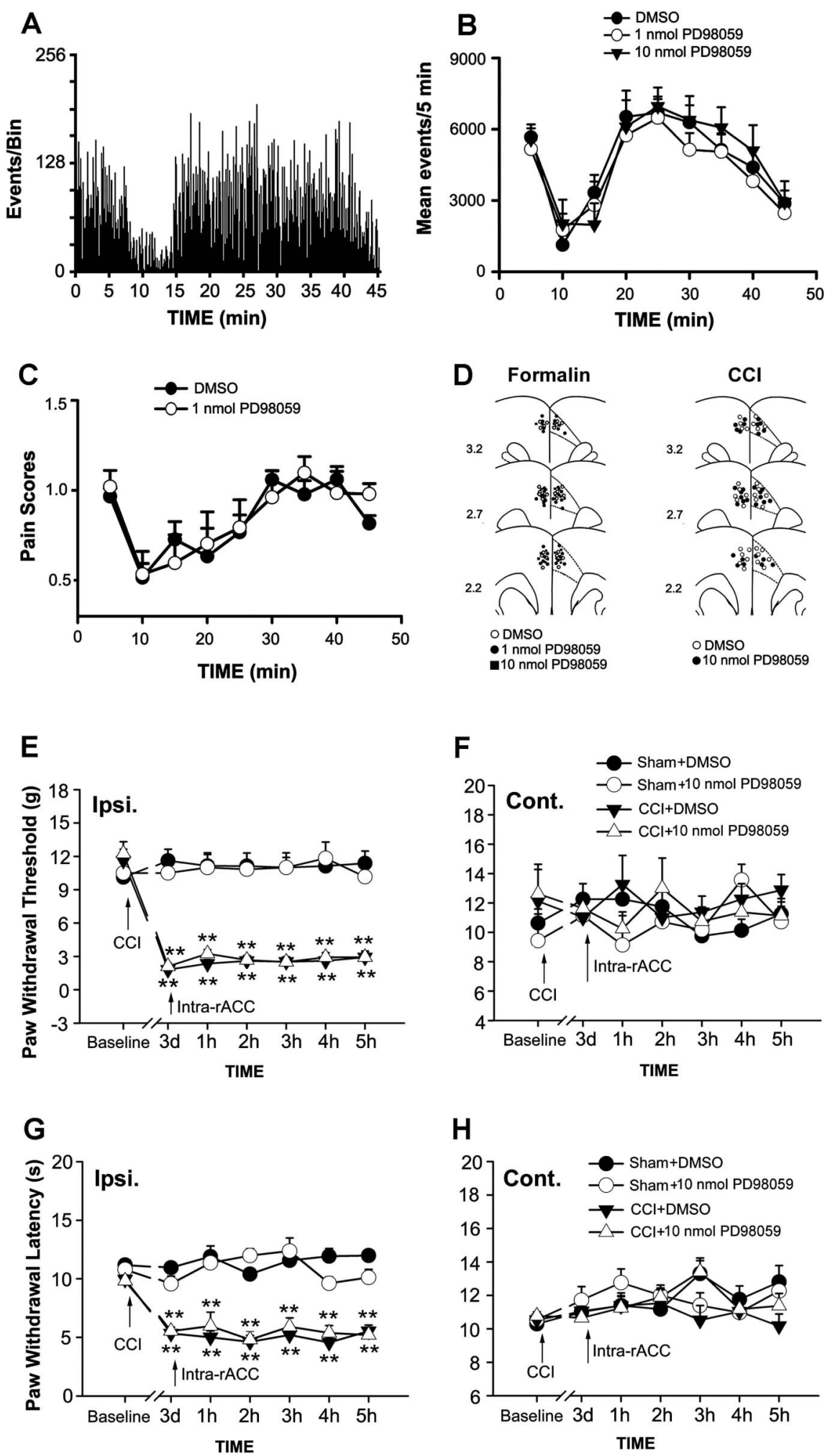

To determine the contribution of ERK in the induction of pain-related aversion, we made bilateral intra-rACC administration of PD98059, a MEK inhibitor that blocks phosphorylation of the ERKs (Alessi et al., 1995), 30 min before F-CPA conditioning (Fig. 2B). PD98059 produced a dose-dependent inhibition of F-CPA acquisition. At doses of 1 and 10 $\mathrm{nmol}$, but not $0.1 \mathrm{nmol}$, bilateral injections of PD98059 significantly blocked F-CPA acquisition. Vehicle (10\% DMSO) had no effect on F-CPA. F-CAP was also blocked by another MEK inhibitor, U0126 at $2 \mathrm{nmol}$ (Fig. 2C). The locations of the PD98059- and U0126-microinjected sites in the rACC are shown in Figure 2D. The MEK inhibitors PD98059 (0.1 nmol, 1 $\mathrm{nmol}$ and $10 \mathrm{nmol}$ ) also dose-dependently inhibited formalin-induced ERK activation in the rACC (Fig. 2E,F).

ERK activation in the spinal cord after formalin injection or spinal nerve ligation (SNL) has been shown to contribute to formalin-induced second phase nociceptive responses (Ji et al., 1999; Karim et al., 2001) and SNL-induced mechanical allodynia (Zhuang et al., 2005). To determine whether ERK activation in the rACC is also important for formalin-induced spontaneous pain-like behavior, we examined formalin-induced biphasic nociceptive responses measured by both automatic movement system (Gao et al., 2004) and manual detection. As shown in Figure $3 A-D$, formalin-induced biphasic nociceptive behavioral responses were not affected by PD98059.

To examine whether ERK activation in the rACC is important for neuropathic pain, we performed another experiment by testing the effect of the MEK inhibitor on neuropathic pain produced by chronic constriction injury (CCI). Consistent with a previous study (Khalil et al., 1999), unilateral CCI operation induced profound thermal hyperalgesia and mechanical allodynia within $3 \mathrm{~d}$ and persisted for weeks in the ipsilateral hindpaw. As shown in Figure 3,E and $G$, CCI-induced hyperalgesia and allodynia were not affected by intrarACC injection of PD98059 (10 nmol). Thus, no detectable differences in PWLs and PWTs were found between DMSOand PD98059-treated groups (Fig. 3E-H).

To determine whether formalininduced ERK activation in the rACC is specific for pain-related aversive learning, we also assessed the effects of MEK inhibitor on general neural processing relating to learning and memory. We first examined the effects of PD98059 on mild foot-shock-induced fear condi-

Figure 3. Inhibition of ERK activation in the rACC does not suppress acute formalin-induced biphasic nociceptive responses and CCl-induced neuropathic pain. $\boldsymbol{A}$-C, Intra-rACC administration of PD98059 has no effect on formalin-induced biphasic nociceptive responses. $A$ is an example of automatic recording of $5 \%$ formalin-induced two-phase nociceptive agitation responses. Bin $=500$ $\mathrm{ms}$. $\boldsymbol{B}$ and $\boldsymbol{C}$ indicate the number of agitation events (automatic recording, $\boldsymbol{B}$ ) and pain scores (manual observation, $\boldsymbol{C}$ ). $n=6-11$. $\boldsymbol{D}$ is the reconstruction of microinjection sites in the bilateral $\mathrm{rACC}$ for formalin and CCI tests. $\boldsymbol{E}-\boldsymbol{H}$, Intra-rACC injection of PD98059 $(10 \mathrm{nmol})$ has no effect on $\mathrm{CCl}$-induced thermal hyperalgesia $(\boldsymbol{E})$ and mechanical allodynia $(\boldsymbol{G})$ in the ipsilateral hindpaw. Neither does PD98059 alter the paw withdrawal latencies (PWLs) and paw withdrawal thresholds (PWTs) in the contralateral hindpaw ( $\boldsymbol{F}$, $\boldsymbol{H}$ ) of CCl rats and in sham surgery rats. PD98059 or vehicle (10\% DMSO) was given on day 3 after $C \mathrm{Cl} .{ }^{* *} p<0.05$ compared with sham $\mathrm{CCl} . n=8$. 
A

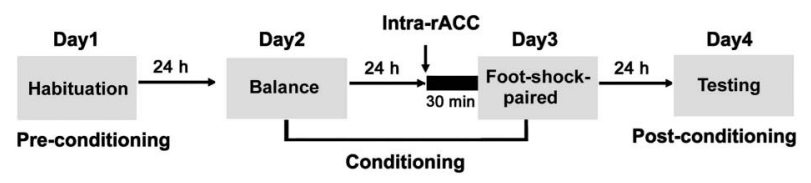

D

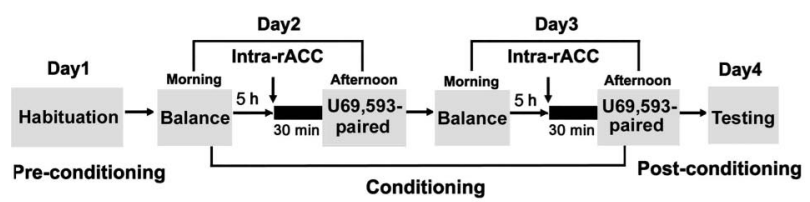

B

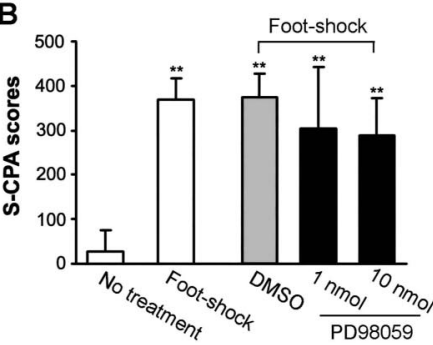

E

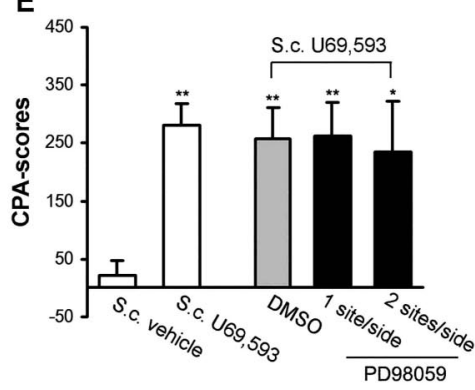

C

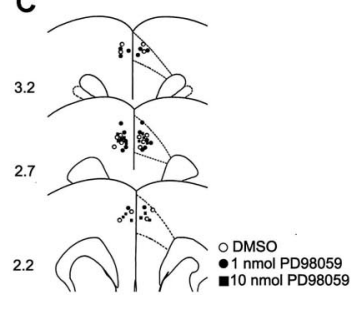

F

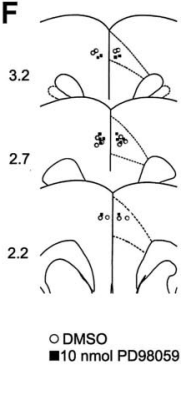

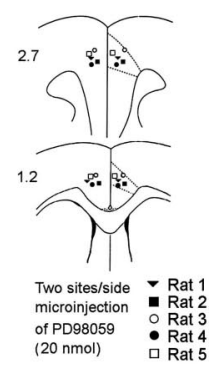

G

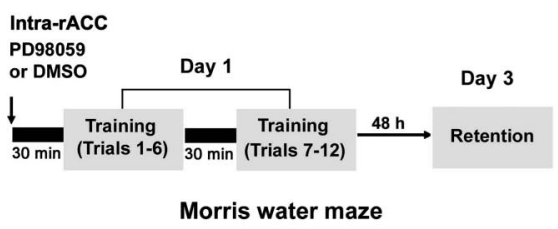

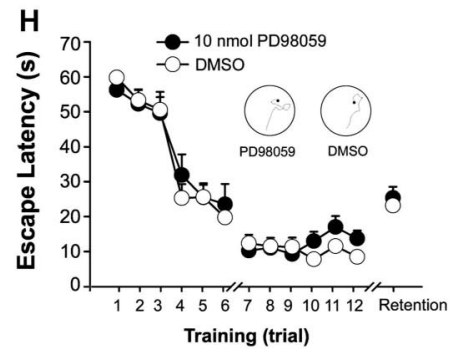

I

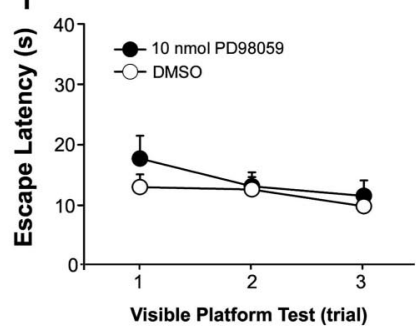

$J$

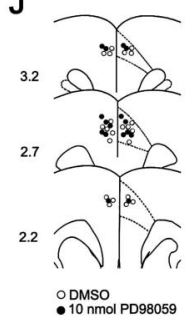

Figure 4. Inhibition of ERK activation in the rACC does not block innocuous stimuli-induced CPA and impair spatial learning and memory in the Morris water maze. A-C, Intra-rACC PD98059 has no significant effect on electric foot-shock (0.5 mA, 2 s)-induced (PA (S-CPA). $\boldsymbol{A}$ shows the protocol for behavioral testing. $\boldsymbol{B}$ shows CPA scores of low intensity S-CPA. $\boldsymbol{C}$ is a reconstruction of microinjection sites of in the bilateral $\mathrm{rACC} .{ }^{* *} p<0.01$ compared with nontreatment control $(n=5-11)$. $\boldsymbol{D}-\boldsymbol{F}$, Intra-rACC infusion of PD98059 dose not inhibit U69,593-induced CPA. D is the protocol for behavioral testing. $E$ shows CPA scores of s.c. injection of U69,593-induced CPA. $F$ is a reconstruction of single (left) and double (right) microinjection sites per side of the rACC. PD98059 was given at a dose of $10 \mathrm{nmol}$ for two sites ( $5 \mathrm{nmol}$ each site) and $20 \mathrm{nmol}$ for four sites ( $5 \mathrm{nmol}$ each site) for both sides of the rACC).** $p<0.01$ compared with vehicle control ( $n=5-11)$. G-J, Intra-rACC injection of PD98059 does not impair spatial learning and memory in the Morris water maze. $\boldsymbol{G}$ is the protocol for behavioral testing. $\boldsymbol{H}$ shows animal's escape latencies to find the submerged platform. Cutoff time was 60 s. PD98059 (10 nmol) or vehicle (10\% DMS0) was given 30 min before training in the Morris water maze with two training sessions of six trials each, and a $30 \mathrm{~min}$ resting period between the two sessions. Memory retention was tested $48 \mathrm{~h}$ after training. The retention values are calculated as the mean of three-trial retention test. Inset, representative swimming traces of a PD98059- and a DMSO-treated rat at $48 \mathrm{~h}$ retention test. $I$, animal's escape latencies to find the visible platform. The platform was raised above the turbid liquid surface to be visible. $n=8-11 . J$ is a reconstruction of microinjection sites of in the bilateral rACC.

tioning (S-CPA). When a low intensity electric foot-shock $(0.5$ $\mathrm{mA}, 2 \mathrm{~s}$ ), which fails to evoke nociceptive responses in spinal dorsal horn neurons (Gao et al., 2004), was paired with a particular compartment in the place conditioning apparatus, CPA was also elicited. However, this S-CPA was not inhibited by intra-rACC injection of PD98059 (Fig. 4A-C). We also examined ERK activation in the rACC after mild foot-shock. Low intensity electric foot-shock $(0.5 \mathrm{~mA}, 2 \mathrm{~s})$ increased pERK levels in the rACC, but this increase was not as robust as that induced by formalin injection (supplemental Figs. 2A, $B$, available at www.jneurosci.org as supplemental material). Unlike formalin-induced wide expression of pERK (laminae IIVI), foot shock induced restricted pERK expression in laminae II-III (supplemental Fig. $2 \mathrm{~A}$, available at www.jneurosci.org as supplemental material), indicating distinct mechanisms may underlie pain-related aversion and fear-related aversion. Interestingly, low intensity foot shock produced a more profound ERK activation than formalin injection in the amygdala, an important brain region for mediating fear conditioning (supplemental Fig. 2C,D, available at www.jneurosci.org as supplemental material). We also tested CPA after a high intensity ( $5 \mathrm{~mA}, 2 \mathrm{~s}$ ) electric foot-shock. Compared with low intensity electric foot-shock, high intensity electric foot-shock produced a higher S-CPA score. Neither was S-CPA by high intensity electric foot-shock inhibited by intra-rACC injection of PD98059 (supplemental Fig. 3A,B, available at www. jneurosci.org as supplemental material).

We next tested another aversive agent U69,593 (0.16 mg/kg), a $\kappa$-opioid receptor agonist (Shippenberg et al., 1993; Johansen et al., 2001). Like formalin injection and electric shock, systemic injection of U69,593 $\left(0.16 \mathrm{mg} / \mathrm{kg}\right.$, s.c.) produced CPA (Fig. $4 D_{-}$ $F)$. Interestingly, U69,593-induced CPA was also ERKindependent, because microinjections of PD98059 into bilateral rACC, either by single-site injection or by double-site injections, had not effect on U69,593-induced CPA (Fig. 4D-F).

In particular, we examined the role of ERK activation in the rACC for spatial learning and memory using Morris water maze. As shown in Fig. 4G-J, both groups of rats receiving PD98059 (10 $\mathrm{nmol}$ ) and vehicle (10\% DMSO) were able to learn to find the submerged platform in the Morris water maze, as the escape la- 
A

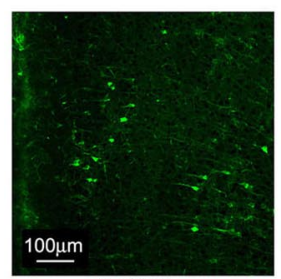

ACSF

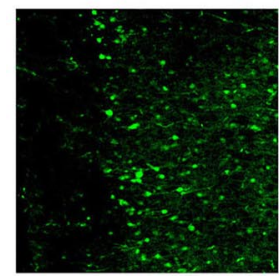

Forskolin

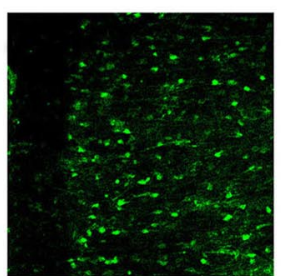
NMDA

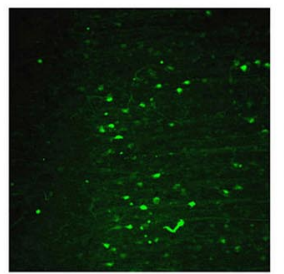

Forskolin+PD98059

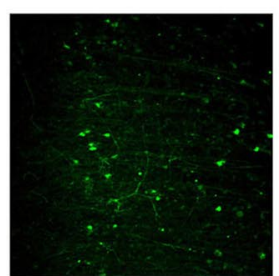

NMDA+APv

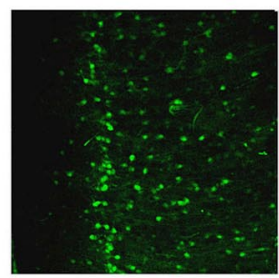

Sp-cAMP
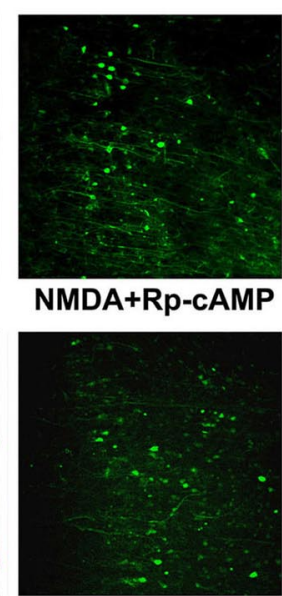

Sp-cAMP+PD98059

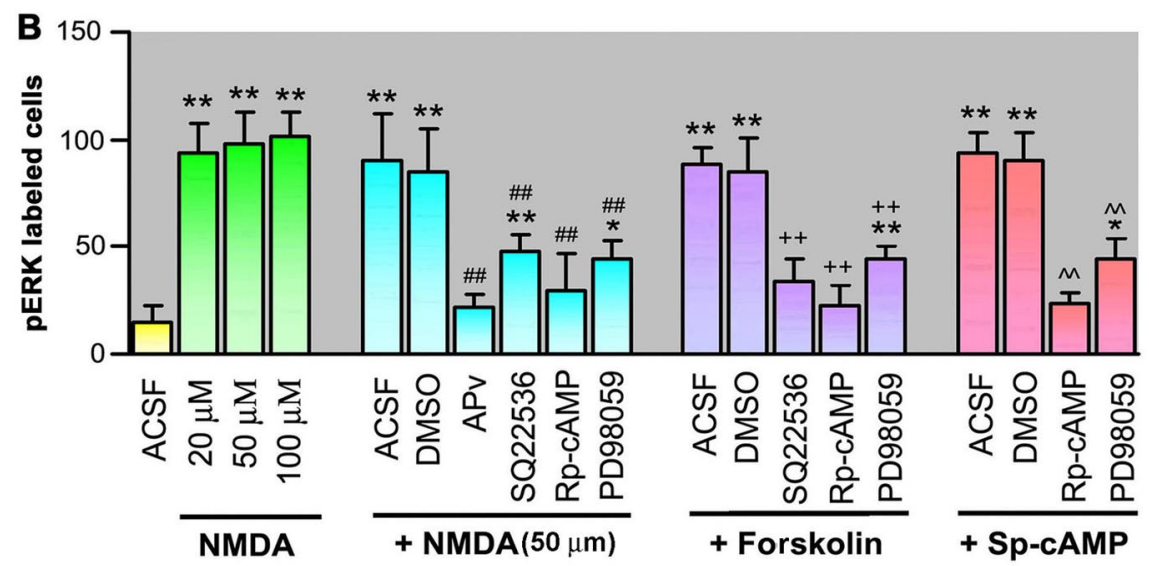

C

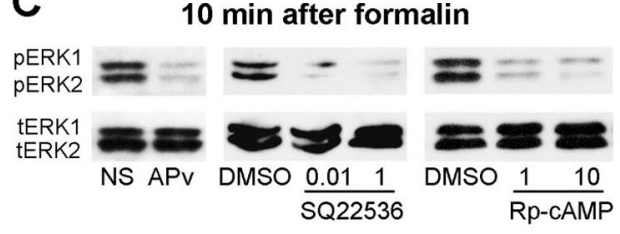

E

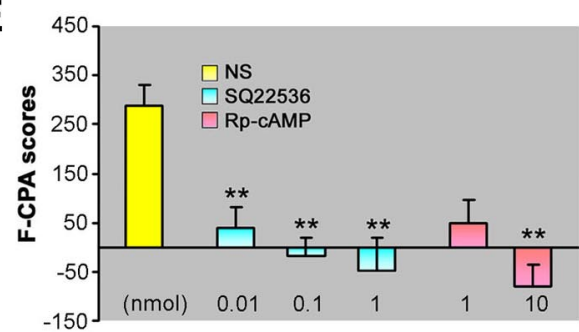

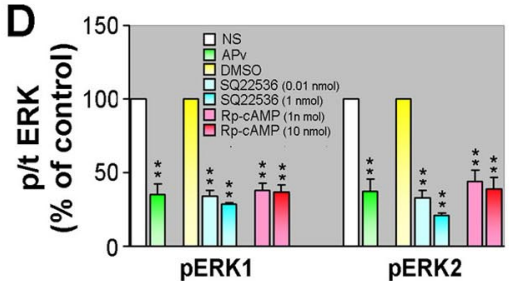

$\mathbf{F}$

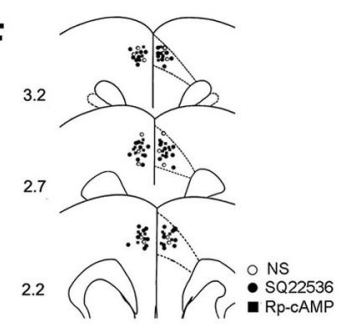

Figure 5. Involvement of CAMP/PKA in NMDA-induced ERK activation in the rACC. $\boldsymbol{A}, \mathrm{pERK}$ immunohistochemistry in rACC slices representative of different treatments. $\boldsymbol{B}$, Numbers of pERK-positive neurons increase after exposure to NMDA $(20,50$, and $100 \mu \mathrm{M})$, forskolin $(50 \mu \mathrm{M})$, and Sp-CAMP (50 $\mu \mathrm{M})$ for $10 \mathrm{~min}$. Preincubation of APv (50 $\mu \mathrm{M}), \mathrm{SQ22536}(50$ $\mu \mathrm{M}), \operatorname{Rp}-\mathrm{CAMP}(50 \mu \mathrm{M})$, or PD98059 $(50 \mu \mathrm{m})$ significantly reduces pERK induced by NMDA $(50 \mu \mathrm{m})$, forskolin, or Sp-cAMP. ${ }^{*} p<0.05$ and ${ }^{* *} p<0.01$ compared with control; ${ }^{\# \#} p<0.01$ compared with NMDA-treated; ${ }^{++} p<0.01$ compared with forskolin-treated; ${ }^{\wedge} p<0.01$ compared with Sp-CAMP treated $(n=6-12)$. C, D, Formalin-induced increases in levels of pERK1 and pERK2 are blocked by intra-rACC preadministration of APv (10 nmol), SQ22536 (0.01, and $1 \mathrm{nmol})$, and Rp-CAMP (1, and $10 \mathrm{nmol}$ ). Both ipsilateral and contralateral sides of the $\mathrm{rACC}$ were pooled together in this experiment. ${ }^{* *} p<0.01$ compared with vehicle (10\% DMSO) control $(n=4-5)$. $\boldsymbol{E}$, Intra-rACC microinjection of AC inhibitor SQ22536 $(0.01,0.1$, and $1 \mathrm{nmol})$ or PKA inhibitor Rp-CAMP $(1$, and $10 \mathrm{nmol})$ before conditioning blocks F-CPA induction. ${ }^{* *} p<0.01$ compared with vehicle (NS) control $(n=6-9)$. $\boldsymbol{F}$, Reconstruction of sites for the microinjections of SQ22536 and Rp-CAMP in the bilateral rACC.

tency became shorter with increased training trials in the both groups (Fig. $4 \mathrm{H}$ ). Accordingly, when tested for memory retention $48 \mathrm{~h}$ after training, the PD98059treated rats also displayed similar escape latency compared with the vehicle controls (Fig. $4 \mathrm{H}$ ). Additionally, no deficit in performance in visible platform test was found in both PD98059- and DMSOtreated groups (Fig. 4I).

To determine whether PD98059 itself is involved in "state-dependent learning" that will result in CPA or conditioned place preference (CPP), intrarACC PD98059 injection was paired with a particular compartment in the place conditioning apparatus without formalin treatment. There were no significant differences between the time spent by rats in the PD98059-paired compartment on the preconditioning and postconditioning days (supplemental Fig. $3 C, D$, available at www.jneurosci. org as supplemental material).

To further determine whether PD98059 has long-lasting effects on future training, the rats that received PD98059 on conditioning day were subsequently retrained without additional drug treatment. Re-training trial was performed $24 \mathrm{~h}$ after the rats were tested CPA in the initial training trial. After retraining, those rats that failed to acquire F-CPA after PD98059 treatment began to develop F-CPA (supplemental Fig. 4, available at www.jneurosci.org as supplemental material).

\section{NMDA receptor is coupled to ERK} activation in the rACC via PKA Bath application of NMDA (20, 50, and $100 \mu \mathrm{M}$ ) for $10 \mathrm{~min}$ produced robust increases in ERK activation in the rACC (Fig. $5 A, B)$, in the presence of $1 \mu \mathrm{M}$ tetrodotoxin (TTX) to block action potential. NMDA-induced ERK activation was completely blocked by the selective NMDA receptor antagonist $\mathrm{APv}(50 \mu \mathrm{M})$ (Fig. 5A). Pretreatment of slices with the PD98059 $(50 \mu \mathrm{M})$ significantly decreased but did not eliminate ERK activation by NMDA (50 $\mu \mathrm{M})$ (Fig. 5B).

To determine whether NMDA receptor is required for peripheral noxious stimulation-evoked ERK activation in the rACC, APv (10 nmol) was microinjected into the bilateral rACC 30 min before intraplantar formalin. Formalin-induced increases in pERK1 and pERK2 levels were prevented by $\mathrm{APv}$ (Fig. 5C,D). Additionally, double immunofluorescence staining indicated that formalin-induced pERK was primarily expressed in neurons that also expressed NMDA receptor NR1 
in the rACC (supplemental Fig. 5A, available at www.jneurosci.org as supplemental material).

A cross talk between PKA and ERK has been reported in the hippocampus and spinal cord (Impey et al., 1998; Roberson et al., 1999; Wong et al., 1999; Hu and Gereau, 2003; Kawasaki et al., 2004; Wei et al., 2006; Sindreu et al., 2007). We sought to determine whether PKA couples NMDA receptor to ERK activation. Preincubation of rACC slices with the AC activator forskolin $(50 \mu \mathrm{M})$ or PKA activator Sp-cAMP $(50 \mu \mathrm{M})$ for $10 \mathrm{~min}$ induced substantial elevation of pERK in the rACC. The forskolin- or Sp-cAMP-induced pERK increase was partially blocked by PD98059 $(50 \mu \mathrm{M})$. Preincubation of rACC slice with the AC inhibitor SQ22536 $(50 \mu \mathrm{M})$ or the PKA inhibitor Rp-cAMPs $(50 \mu \mathrm{M})$ prevented ERK activation by forskolin, SpcAMP, or NMDA (Fig. 5A,B). The role of cAMP/PKA in ERK activation was also confirmed in intact animals: intra-rACC injection of SQ22536 ( 0.01 and $1 \mathrm{nmol})$ or Rp-cAMP (1 and $10 \mathrm{nmol}$ ), $30 \mathrm{~min}$ before formalin stimulation, significantly attenuated ERK activation in the rACC (Fig. $5 C, D)$. Consistently, we observed a robust activation of PKA in the rACC after formalin injection, using an antibody that recognized pPKA RII (Ser 96) to monitor PKA activation (supplemental Fig. 5B, available at www.jneurosci.org as supplemental material).

To investigate the role of PKA in the development of pain-related negative emotion, we further investigated the effects of blocking PKA activation on F-CPA. Both AC inhibitor SQ22536 (0.01, 0.1 and $1 \mathrm{nmol}$ ) and PKA inhibitor RpcAMP ( 1 and $10 \mathrm{nmol}$ ) blocked F-CPA acquisition (Fig. 5E). To rule out SQ22536 and Rp-cAMP have long-lasting effects on future training, the rats that received SQ22536 or Rp-cAMP on conditioning day were subsequently retrained without additional drug treatment. Re-training trial was performed $24 \mathrm{~h}$ after the rats were tested CPA in the initial training trial. After retraining, those rats that failed to acquire F-CPA after SQ22536 or RpcAMP treatment began to develop F-CPA (supplemental Fig. 4, available at www.jneurosci.org as supplemental material).

ERK activation mediates CREB phosphorylation in the rACC To determine the downstream mechanisms by which ERK mediates affective pain, we examined the activation of the transcription factor CREB, since ERK could facilitate gene expression via phosphorylating the CREB (Arthur et al., 2004). In nontreated rACC slices, there is basal pCREB expression. NMDA $(50 \mu \mathrm{M})$ exposure induced a significant increase in pCREB expression in rACC neurons. pCREB was also induced by bath application of the AC activator forskolin $(50 \mu \mathrm{M})$ or the PKA activator SpcAMP $(50 \mu \mathrm{M})$. Preincubation with PD98059 (50 $\mu \mathrm{M})$ significantly suppressed CREB phosphorylation by NMDA, forskolin, or Sp-cAMP. Accordingly, AC inhibitor SQ22536 (50 $\mu \mathrm{M})$ and PKA inhibitor Rp-cAMP $(50 \mu \mathrm{M})$ also reduced CREB phosphorylation by these activators (Fig. 6A,B). Double immunofluorescence staining indicated that $\sim 96 \%$ of pERK-positive cells (196 pERK/pCREB double-labeled cells out of 205 pERK singlelabeled in 4 ACC sections from 3 rats), were also pCREB-positive. And further, pCREB was exclusively expressed in neurons in the rACC (Fig. 6A).

ERK activation also induced pCREB in vivo. Formalin injection increased pCREB levels in the bilateral rACC, with a time course similar to that of pERK, reaching a peak at $30 \mathrm{~min}$ and maintained at elevated levels for $24 \mathrm{~h}$ (Fig. $7 A-D$ ). Double immunofluorescence staining indicated that $\sim 95 \%$ of pERKpositive cells are also pCREB-positive $10 \mathrm{~min}$ after formalin injection (243 pERK/pCREB double-labeled cells out of 265 pERK single-labeled in 6 ACC sections from 2 rats) (Fig. 7A) and further, pCREB was colocalized with Fos-positive nucleus in the rACC (supplemental Fig. 5C, available at www.jneurosci.org as supplemental material). Intra-rACC pretreatment of PD98059 

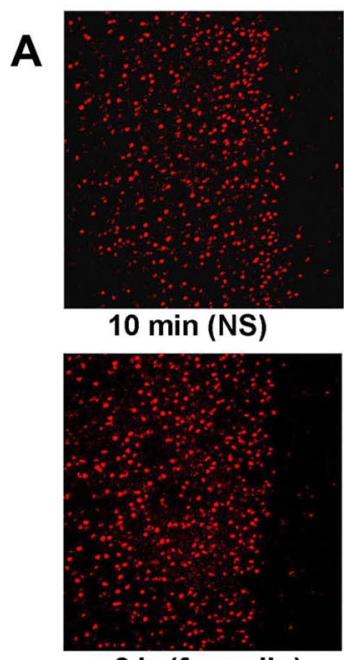

$2 \mathrm{~h}$ (formalin)

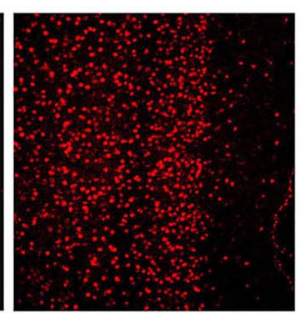

$10 \mathrm{~min}$ (formalin)

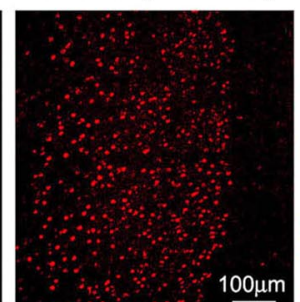

$24 \mathrm{~h}$ (formalin)

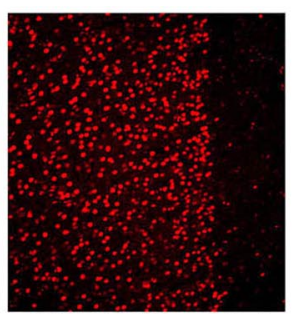

30 min (formalin)

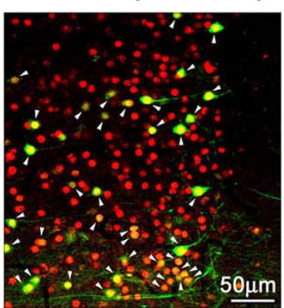

pERK/pCREB
C
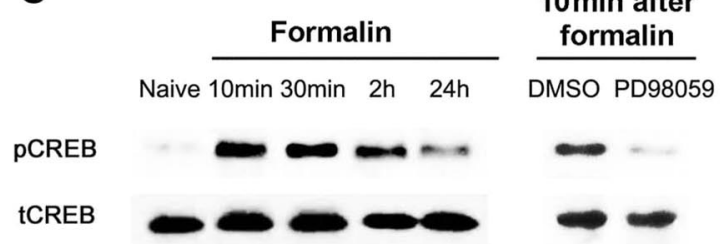

Ipsi.

pCREB

tCREB

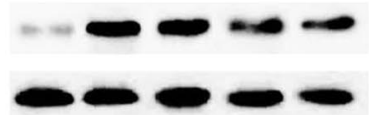

Cont.
B

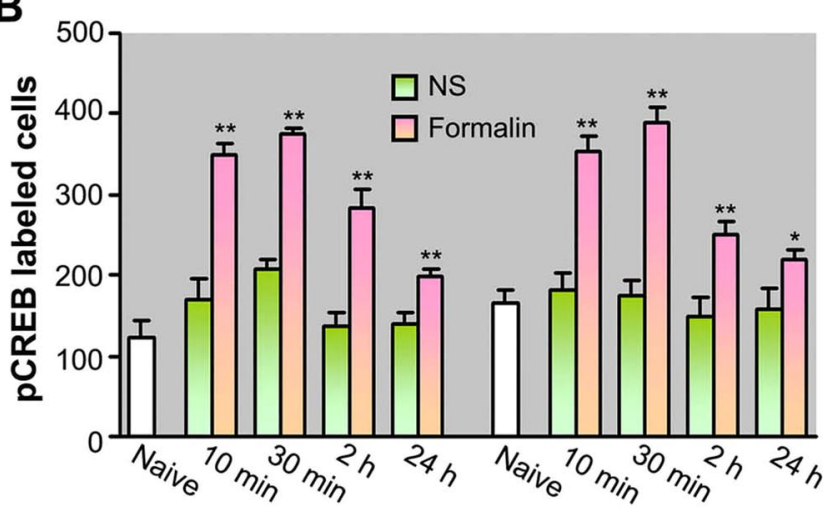

D

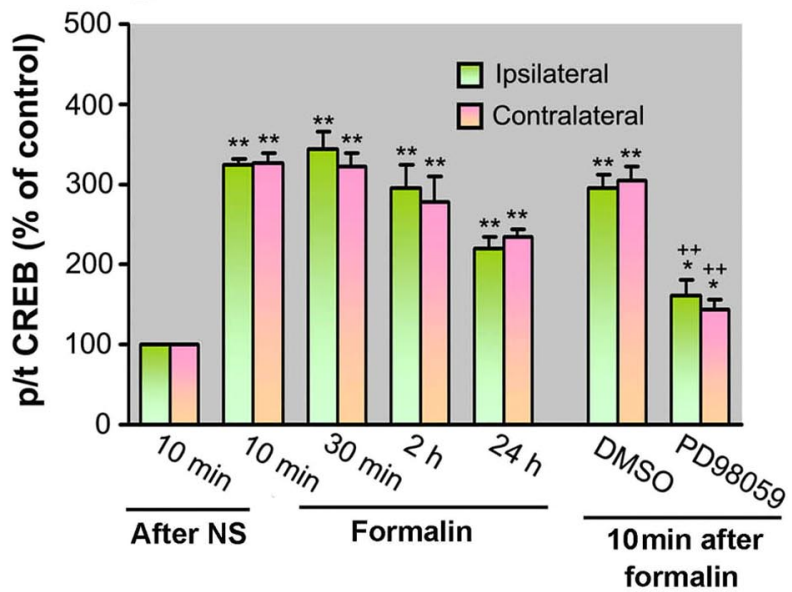

Figure 7. Time course of formalin-induced CREB phosphorylation in the bilateral rACC. $A$, pCREB immunohistochemistry in the contralateral rACC from coronal brain sections representative of NS injection at $10 \mathrm{~min}$ and formalin injection at $10 \mathrm{~min}, 30 \mathrm{~min}, 2 \mathrm{~h}$, and $24 \mathrm{~h}$. Low right, double immunofluroescence reveals that formalin-induced pERK (green) colocalizes with pCREB (red) in vivo. Arrowheads indicate double-labeled cells. $\boldsymbol{B}$, numbers of $\mathrm{pCREB}$-positive neurons in the bilateral rACC are significantly greater in formalin-treated rats than that of NS controls. $\boldsymbol{C}, \boldsymbol{D}$, Western blot analysis reveals an increase in the $p C R E B$ levels in the rACC after formalin injection, which is blocked by intra-rACC preadministration of PD98059 $(10 \mathrm{nmol}) .{ }^{*} p<0.05$, ${ }^{* *} p<0.01$ compared with control; ${ }^{++} p<0.01$ compared with DMSO pretreatment $(n=5)$.

(10 nmol, $30 \mathrm{~min}$ before formalin injection) partially inhibited pCREB induction at $10 \mathrm{~min}$ after formalin injection (Fig. 7C,D). The vehicle (10\% DMSO) had no effect on formalin-induced pCREB.

ERK activation in the rACC is required for the expression of pain-related aversion

Our results have indicated that ERK activation in the rACC is necessary for the acquisition of F-CPA (Fig. 2). To investigate whether ERK activation is also required for the expression of F-CPA, we administered the MEK inhibitor U0126 (2 nmol) into the bilateral rACC 0.5 or $6 \mathrm{~h}$ before CPA test on postconditioning day (Fig. $8 \mathrm{~A}$ ). As expected, vehicle (35\% DMSO)-treated rats showed higher CPA scores. In contrast, the U0126-treated rats, either $30 \mathrm{~min}$ or $6 \mathrm{~h}$ before testing, did not show any significant avoidance in formalinpaired compartment (Fig. $8 \mathrm{~B}$ ). Thus, inhibition of ERK pathway before re-exposure to the conditioning context resulted in impairment in the expression of pain-related aversion.

To investigate whether the pain experience retrieval activates ERK in the rACC during the expression of pain-related aversion, rats were re-exposed to the conditioning context (formalinpaired compartment) for 10 min on day 4 of the F-CPA paradigm (Fig. 8C). A significant activation of ERK in the rACC was observed at $10 \mathrm{~min}$ and $30 \mathrm{~min}$, but this activation recovered at $6 \mathrm{~h}$ after retrieval (re-exposure to the conditioning context) (Fig. $8 D-F, H)$. This retrieval also increased pCREB levels in the rACC (Fig. 8G,I). The induction of both pERK and pCREB was markedly reduced by U0126 (Fig. $8 F-I$ ).

\section{Discussion}

ERK activation in the spinal cord is required for nociceptive pain sensitization in the peripheral inflammation models (Ji et al., 1999; 2002; Karim et al., 2001; Adwanikar et al., 2004; Cruz et al., 2005). The present study has further shown that intraplantar formalin injection also activates ERK in the rACC and this activation is critical for the induction and expression of affective pain.

\section{ERK activation in the rACC and affective pain}

Intradermal injection of diluted formalin is painful in human (Dubuisson and Dennis, 1977; Puig and Sorkin, 1996). The fact 
that formalin injection produces both CPA and other nociceptive behaviors in animals indicates that formalin is aversive to animals in a manner resembling the response to noxious stimuli in humans (Johansen et al., 2001). Our study showed that formalin injection induced a persistent ERK activation $(>24 \mathrm{~h})$ in laminae II-III and a rapid transient ERK activation $(\leq 30 \mathrm{~min}$ ) in deep layers (laminae V-VI) of the bilateral rACC. This pERK expression pattern is different from a recent report showing a transient ERK activation (within $90 \mathrm{~min}$ ) in lamina II neurons in the ACC after formalin injection (Wei and Zhuo, 2008). This discrepancy may result from differences in time course ( $24 \mathrm{~h}$ vs 90 min) and subregions of the ACC. Actually, formalin-induced pERK in the rACC was located in all layers, whereas formalininduced $\mathrm{pERK}$ in the cACC was primarily limited in laminae II/III (Fig. 1). Further, pERK was induced predominantly in pyramidal neurons of the rACC. The distribution of pERK in the ACC is similar to those neurons in the ACC that respond to noxious stimulation. A large number of neurons within laminae III and $\mathrm{V}$ of the ACC receive noxious input from wide areas of the body bilaterally (Sikes and Vogt, 1992; Yamamura et al., 1996). Most nociceptive neurons are classified as pyramidal neurons and found in rACC (Yamamura et al., 1996). Increasing evidence indicates an important role of the rACC in painrelated negative affect (Rainville et al., 1997; Johansen et al., 2001; Kung et al., 2003; Gao et al., 2004). Like c-Fos expression (Lei et al. 2004a,b), ERK activation in the rACC may serve as a marker for the activation of rACC neurons under an affective pain condition. Moreover, ERK activation in the rACC is required for the development of affective pain, because intra-rACC of MEK inhibitor PD98059 or U0126 prevented the induction of F-CPA. We further demonstrated that activation of ERK in the rACC is required for the expression of pain-related aversion. Consistently, a robust pERK increase was also found in the rACC after pain retrieval. Studies from Izquierdo's laboratory have shown that retrieval of memory requires the activation of the PKA and ERK signaling cascades in several brain regions including the ACC (Barros et al., 2000, 2001, 2003; Izquierdo et al., 2001).

\section{ERK activation in the rACC and nociceptive pain}

Our data showed that intra-rACC injection of PD98059 did not suppress formalin-induced nociceptive pain, in support of earlier studies from our and other groups showing that either bilateral lesions of the ACC or blockade of glutamate receptors in the
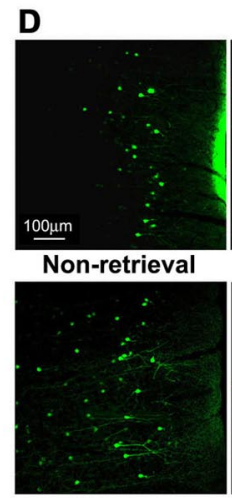

$30 \mathrm{~min}$
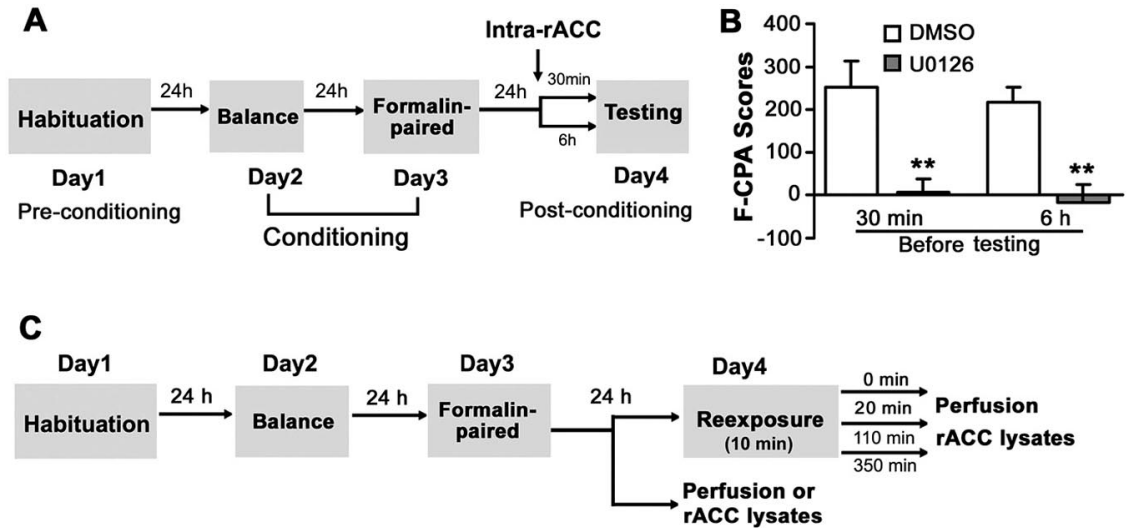

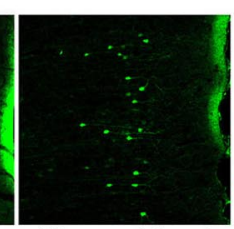

Sham retrieval

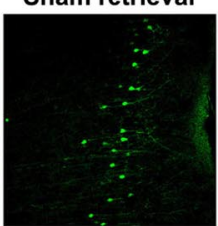

$2 \mathrm{~h}$
E

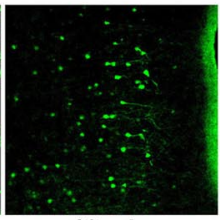

$10 \mathrm{~min}$

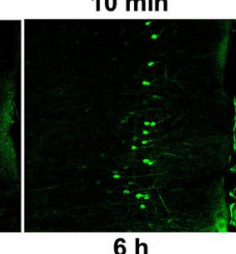

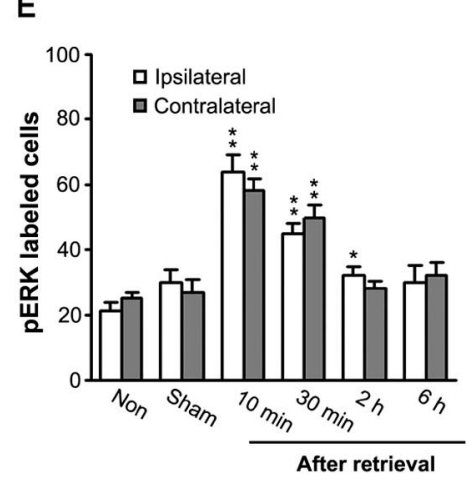

F

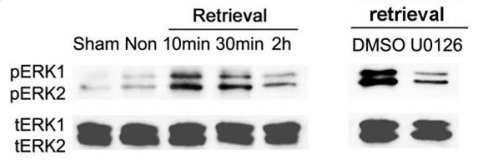

H

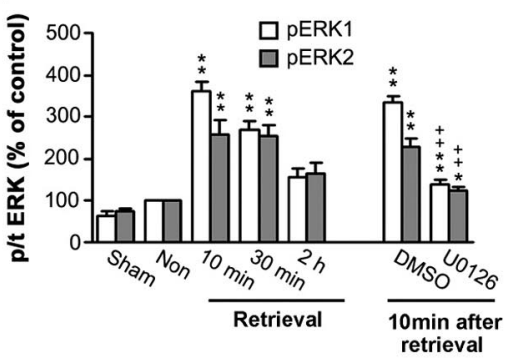

G

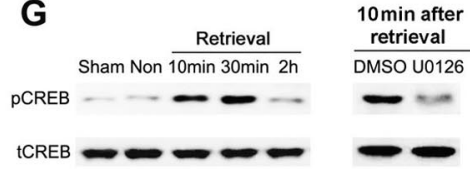

Figure 8. ERK activation in the $\mathrm{rACC}$ is required for the expression of F-CPA. $\boldsymbol{A}$, Schematic demonstration of behavioral testing. $\boldsymbol{B}$, Intra-rACC microinjection of MEK inhibitor U0126 (2 nmol) before test blocks F-CPA expression. ${ }^{* *} p<0.01$ compared with vehicle (35\% DMSO) control ( $n=8-11)$. C, Schematic of experimental design used in the following studies. D-I, Time course of F-CPA retrieval-induced $p E R K$ and $p C R E B$ increases in the $r A C C$. Immunohistochemistry for $p E R K(\boldsymbol{D}, \boldsymbol{E})$ and Western blot for $p$ ERK1 and pERK2 $(\boldsymbol{F}, \boldsymbol{H})$ as well as pCREB $(\boldsymbol{G}, \boldsymbol{I})$ from the rACC show significant increases in the levels of $p$ ERK and $p$ CREB after F-CPA retrieval, and all the increases are blocked by intra-rACC injection of MEK inhibitor U0126, given 30 min before retrieval. ${ }^{*} p<$ $0.05,{ }^{* *} p<0.01$ compared with nonretrieval; ${ }^{\# \#} p<0.01$ compared with vehicle (35\% DMSO) control $(n=5)$.

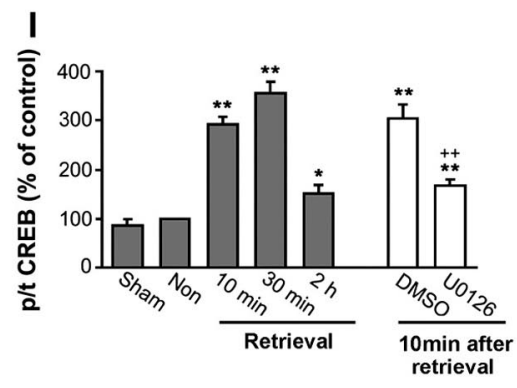

rACC do not inhibit formalin-induced nociceptive responses (Johansen et al., 2001; Gao et al., 2004; Johansen and Fields, 2004; Lei et al., 2004a; Ren et al., 2006). Furthermore, we did not observe any effect of blocking rACC ERK activation on CCIinduced neuropathic pain (Fig. 3), consistent with a report from LaGraize et al. that ACC lesion in animals with L5 spinal nerve ligation (SNL) did not alter mechanical hypersensitivity (LaGraize et al., 2004). In contrast, Wu et al. (2005) reported that 
intra-ACC injection of a selective NR2B antagonist, Ro25-6981, produced a transient inhibition $(30 \mathrm{~min})$ of CFA-induced inflammatory pain in mice. This discrepancy may be due to differences in species of animals (rats vs mice), phases of pain (early vs late), and types of injury (inflammation vs nerve injury). It appears that the ACC does not modulate neuropathic pain (SNL and CCI), acute pain [e.g., tail-flick reflex, hot plate response (Wu et al., 2005)], and formalin-induced spontaneous pain.

Recently, Carrasquillo and Gereau (2007) demonstrated that ERK activation in the amygdala is necessary and sufficient for nociceptive pain sensitization. Also, previous studies from our (Gao et al., 2004) and other groups (Tanimoto et al., 2003) demonstrated that bilateral lesions of the amygdala blocked F-CPA induction. Considering the important role of the amygdala in pain and negative affect (Neugebauer et al., 2004, 2007), and reciprocal connections between the amygdala and ACC (McDonald and Mascagni, 1996), it is likely that the neural network of the two structures is critical for the negative affect of pain.

\section{ERK activation in the $\mathrm{ACC}$ and aversive learning}

Given that F-CPA paradigm is based on the associative learning between painful stimulus-induced aversive emotion and neutral environmental context, inhibition of F-CPA by MEK inhibitor in the rACC may be caused by a disruption in neural processing related to learning and memory. Although we cannot completely rule out this possibility, it is important to point out that neither was the animal's ability to acquire CPA induced by nonnociceptive aversive stimuli, nor spatial learning/memory in Morris water maze testing impaired by intra-rACC injection of PD98059. Consistently, previous findings from our and other groups indicate that CPA induced either by foot-shock (Gao et al., 2004) or U69,593 (Johansen et al., 2001) was not blocked by ACC lesions. Further, morphine- and cocaine-induced CPP (conditioned place preference) (Tzschentke and Schmidt, 1998a,b) is not blocked by ACC lesions either. Thus, the ERK pathway in the rACC is particularly important for pain-related negative emotion and pain-related aversive learning rather than general learning and memory. It is possible that the perception of pain-related negative affect and pain-related aversive learning are not clearly separable at the neural level. ERK activation in the rACC might be not only necessary for perception of pain-related affect, but also be essential for the learning that underlies recognition of pain-predictive cues and avoidance. As a support, electrophysiological studies have demonstrated that ACC neurons respond both to noxious stimuli and cues that predict a noxious stimulus (Koyama et al., 1998; Hutchison et al., 1999).

\section{Upstream mechanisms causing ERK activation in the rACC}

NMDA receptor is widely implicated in ERK activation (Finkbeiner and Greenberg, 1996; Xia et al., 1996; English and Sweatt, 1997; Krapivinsky et al., 2003; Ji et al., 2007). $\mathrm{Ca}^{2+}$ influx via NMDA receptor is essential for ERK activation (Lever et al., 2003; Sindreu et al., 2007). $\mathrm{Ca}^{2+}$ can elevate intracellular cAMP levels leading to the activation of PKA. PKA then activates ERK pathway through Rap1, then B-Raf (Impey et al., 1999). Our data showed that NMDA perfusion of brain slices induced a robust pERK increase in the rACC, and formalin-induced pERK was almost completely blocked by APv. Further, both AC and PKA activators elevated pERK to similar levels as NMDA. Inhibition of cAMP/PKA significantly blocked F-CPA and ERK activation by NMDA in vitro and by formalin in vivo (Fig. 5). These data strongly imply the NMDA receptor-cAMP-PKA-ERK pathway in the rACC for the control of affective pain. Consistently, Sindreu et al. have demonstrated that activation of ERK by cAMP/PKA and $\mathrm{Ca}^{2+}$ in the hippocampus is critical for synaptic plasticity and long-term memory (Sindreu et al., 2007). However, a very recent study showed that U0126 did not inhibit synaptic facilitation by forskolin in the amygdala, suggesting that PKA and ERK in the amygdala modulate pain-related synaptic plasticity through separate signaling pathways (Fu et al., 2008). Thus, the PKA-ERK pathway may only apply to certain brain areas.

\section{Downstream mechanisms underlying ERK's action in the rACC}

ERK activation has been implicated in CREB-mediated gene transcription in cortical, hippocampal, and spinal cord neurons (Impey et al., 1998; Vanhoutte et al., 1999; Ji et al., 2002; Kawasaki et al., 2004). We found that nociceptive conditioning-induced ERK activation in the rACC is also coupled to CREB phosphorylation. pERK was largely colocalized with pCREB in the same rACC neurons after formalin injection (Fig. 7A). Furthermore, the upstream cascade that activated ERK, such as the NMDA receptor-cAMP-PKA cascade, also caused CREB phosphorylation in the rACC slices. However, target genes that are regulated by CREB-mediated transcription in the rACC and the role of these genes in pain affect remain to be investigated, although c-fos is induced in the rACC by formalin injection and retrieval of nociceptive experience (Liu et al., 1998; Lei et al., 2004a,b) and pCREB and Fos are colocalized in the same rACC neurons (supplemental Fig. 5C, available at www.jneurosci.org as supplemental material).

In addition to nuclear localization, we found pERK in the cytoplasm and dendrites of pyramidal neurons (Fig. $1 \mathrm{~A}$ ), indicating a nontranscriptional role of ERK. Indeed, ERK-mediated posttranslational regulation has been suggested in the induction of central sensitization (Ji et al., 1999; Fukui et al., 2007). Remarkably, ERK activation potentiates glutamatergic synaptic transmission to produce pain hypersensitivity (Kohno et al., 2008) and increases excitability of dorsal horn neurons by suppressing Kv4.2-mediated A-type $\mathrm{K}^{+}$currents (Hu et al., 2007). It remains to be investigated whether these ERK-mediated mechanisms also work in ACC neurons.

\section{References}

Adwanikar H, Karim F, Gereau RW 4th (2004) Inflammation persistently enhances nocifensive behaviors mediated by spinal group I mGluRs through sustained ERK activation. Pain 111:125-135.

Alessi DR, Cuenda A, Cohen P, Dudley DT, Saltiel AR (1995) PD 098059 is a specific inhibitor of the activation of mitogen-activated protein kinase kinase in vitro and in vivo. J Biol Chem 270:27489-27494.

Arthur JS, Fong AL, Dwyer JM, Davare M, Reese E, Obrietan K, Impey S (2004) Mitogen- and stress-activated protein kinase 1 mediates cAMP response element-binding protein phosphorylation and activation by neurotrophins. J Neurosci 24:4324-4332.

Bailey DJ, Kim JJ, Sun W, Thompson RF, Helmstetter FJ (1999) Acquisition of fear conditioning in rats requires the synthesis of mRNA in the amygdala. Behav Neurosci 113:276-282.

Barros DM, Izquierdo LA, Mello e Souza T, Ardenghi PG, Pereira P, Medina JH, Izquierdo I (2000) Molecular signaling pathways in the cerebral cortex are required for retrieval of one-trial avoidance learning in rats. Behav Brain Res 114:183-192.

Barros DM, Mello e Souza T, de Souza MM, Choi H, DeDavid e Silva T, Lenz G, Medina JH, Izquierdo I (2001) LY294002, an inhibitor of phosphoinositide 3-kinase given into rat hippocampus impairs acquisition, consolidation and retrieval of memory for one-trial step-down inhibitory avoidance. Behav Pharmacol 12:629-634.

Barros DM, Izquierdo LA, Medina JH, Izquierdo I (2003) Pharmacological findings contribute to the understanding of the main physiological mechanisms of memory retrieval. Curr Drug Targets CNS Neurol Disord 2:81-94. 
Bennett GJ, Xie YK (1988) A peripheral mononeuropathy in rat that produces disorders of pain sensation like those seen in man. Pain 33:87-107.

Bozon B, Kelly A, Josselyn SA, Silva AJ, Davis S, Laroche S (2003) MAPK, CREB and zif268 are all required for the consolidation of recognition memory. Philos Trans R Soc Lond B Biol Sci 358:805-814.

Carrasquillo Y, Gereau RW 4th (2007) Activation of the extracellular signalregulated kinase in the amygdala modulates pain perception. J Neurosci 27:1543-1551.

Crombez G, Vlaeyen JW, Heuts PH, Lysens R (1999) Pain-related fear is more disabling than pain itself: evidence on the role of pain-related fear in chronic back pain disability. Pain 80:329-339.

Cruz CD, Avelino A, McMahon SB, Cruz F (2005) Increased spinal cord phosphorylation of extracellular signal-regulated kinases mediates micturition overactivity in rats with chronic bladder inflammation. Eur J Neurosci 21:773-781.

Dubuisson D, Dennis SG (1977) The formalin test: a quantitative study of the analgesic effects of morphine, meperidine, and brain stem stimulation in rats and cats. Pain 4:161-174.

English JD, Sweatt JD (1996) Activation of p42 mitogen-activated protein kinase in hippocampal long term potentiation. J Biol Chem 271:24329-24332.

English JD, Sweatt JD (1997) A requirement for the mitogen-activated protein kinase cascade in hippocampal long term potentiation. J Biol Chem 272:19103-19106.

Finkbeiner S, Greenberg ME (1996) $\mathrm{Ca}(2+)$-dependent routes to Ras: mechanisms for neuronal survival, differentiation, and plasticity? Neuron 16:233-236.

Fu Y, Han J, Ishola T, Scerbo M, Adwanikar H, Ramsey C, Neugebauer V (2008) PKA and ERK, but not PKC, in the amygdala contribute to painrelated synaptic plasticity and behavior. Mol Pain 4:26.

Fukui T, Dai Y, Iwata K, Kamo H, Yamanaka H, Obata K, Kobayashi K, Wang S, Cui X, Yoshiya S, Noguchi K (2007) Frequency-dependent ERK phosphorylation in spinal neurons by electric stimulation of the sciatic nerve and the role in electrophysiological activity. Mol Pain 3:18.

Gao YJ, Ren WH, Zhang YQ, Zhao ZQ (2004) Contributions of the anterior cingulate cortex and amygdala to pain- and fear-conditioned place avoidance in rats. Pain 110:343-353.

Hu HJ, Gereau RW 4th (2003) ERK integrates PKA and PKC signaling in superficial dorsal horn neurons. II. Modulation of neuronal excitability. J Neurophysiol 90:1680-1688.

Hu HJ, Alter BJ, Carrasquillo Y, Qiu CS, Gereau RW 4th (2007) Metabotripic glutamate receptor 5 modulates nociceptive plasticity via extracellular signal-regulated kinase-Kv4.2 signaling in spinal cord dorsal horn neurons. J Neurosci 27:13181-13191.

Hutchison WD, Davis KD, Lozano AM, Tasker RR, Dostrovsky JO (1999) Pain-related neurons in the human cingulate cortex. Nat Neurosci 2:403-405.

Impey S, Obrietan K, Wong ST, Poser S, Yano S, Wayman G, Deloulme JC, Chan G, Storm DR (1998) Cross talk between ERK and PKA is required for $\mathrm{Ca} 2+$ stimulation of CREB-dependent transcription and ERK nuclear translocation. Neuron 21:869-883.

Impey S, Obrietan K, Storm DR (1999) Making new connections: role of ERK/MAP kinase signaling in neuronal plasticity. Neuron 23:11-14.

Izquierdo LA, Viola H, Barros DM, Alonso M, Vianna MR, Furman M, Levi de Stein M, Szapiro G, Rodrigues C, Choi H, Medina JH, Izquierdo I (2001) Novelty enhances retrieval: molecular mechanisms involved in rat hippocampus. Eur J Neurosci 13:1464-1467.

Jett MF, Michelson S (1996) The formalin test in rat: validation of an automated system. Pain 64:19-25.

Ji RR, Baba H, Brenner GJ, Woolf CJ (1999) Nociceptive-specific activation of ERK in spinal neurons contributes to pain hypersensitivity. Nat Neurosci 2:1114-1119.

Ji RR, Befort K, Brenner GJ, Woolf CJ (2002) ERK MAP kinase activation in superficial spinal cord neurons induces prodynorphin and NK-1 upregulation and contributes to persistent inflammatory pain hypersensitivity. J Neurosci 22:478-485.

Ji RR, Kohno T, Moore KA, Woolf CJ (2003) Central sensitization and LTP: do pain and memory share similar mechanisms? Trends Neurosci 26:696-705.

Ji RR, Kawasaki Y, Zhuang ZY, Wen YR, Zhang YQ (2007) Protein kinases as potential targets for the treatment of pathological pain. Handb Exp Pharmacol 177:359-389.
Jin M, Wang XM, Tu Y, Zhang XH, Gao X, Guo N, Xie Z, Zhao G, Jing N, Li BM, Yu L (2005) The negative cell cycle regulator, Tob (transducer of ErbB-2), is a multifunctional protein involved in hippocampusdependent learning and memory. Neuroscience 131:647-659.

Johansen JP, Fields HL (2004) Glutamatergic activation of anterior cingulate cortex produces an aversive teaching signal. Nat Neurosci 7:398-403.

Johansen JP, Fields HL, Manning BH (2001) The affective component of pain in rodents: direct evidence for a contribution of the anterior cingulate cortex. Proc Natl Acad Sci U S A 98:8077-8082.

Karim F, Wang CC, Gereau RW 4th (2001) Metabotropic glutamate receptor subtypes 1 and 5 are activators of extracellular signal-regulated kinase signaling required for inflammatory pain in mice. J Neurosci 21:3771-3779.

Kawasaki Y, Kohno T, Zhuang ZY, Brenner GJ, Wang H, Van Der Meer C, Befort K, Woolf CJ, Ji RR (2004) Ionotropic and metabotropic receptors, protein kinase $\mathrm{A}$, protein kinase $\mathrm{C}$, and Src contribute to $\mathrm{C}$-fiberinduced ERK activation and cAMP response element-binding protein phosphorylation in dorsal horn neurons, leading to central sensitization. J Neurosci 24:8310-8321.

Khalil Z, Liu T, Helme RD (1999) Free radicals contribute to the reduction in peripheral vascular responses and the maintenance of thermal hyperalgesia in rats with chronic constriction injury. Pain 79:31-37.

Kohno T, Wang H, Amaya F, Brenner GJ, Cheng JK, Ji RR, Woolf CJ (2008) Bradykinin enhances AMPA and NMDA receptor activity in spinal cord dorsal horn neurons by activating multiple kinases to produce pain hypersensitivity. J Neurosci 28:4533-4540.

Koyama T, Tanaka YZ, Mikami A (1998) Nociceptive neurons in the macaque anterior cingulate activate during anticipation of pain. Neuroreport 9:2663-2667.

Krapivinsky G, Krapivinsky L, Manasian Y, Ivanov A, Tyzio R, Pellegrino C, Ben-Ari Y, Clapham DE, Medina I (2003) The NMDA receptor is coupled to the ERK pathway by a direct interaction between NR2B and RasGRF1. Neuron 40:775-784.

Kung JC, Su NM, Fan RJ, Chai SC, Shyu BC (2003) Contribution of the anterior cingulate cortex to laser-pain conditioning in rats. Brain Res 970:58-72.

LaGraize SC, Labuda CJ, Rutledge MA, Jackson RL, Fuchs PN (2004) Differential effect of anterior cingulate cortex lesion on mechanical hypersensitivity and escape/avoidance behavior in an animal model of neuropathic pain. Exp Neurol 188:139-148.

Lei LG, Sun S, Gao YJ, Zhao ZQ, Zhang YQ (2004a) NMDA receptors in the anterior cingulate cortex mediate pain-related aversion. Exp Neurol 189:413-421.

Lei LG, Zhang YQ, Zhao ZQ (2004b) Pain-related aversion and Fos expression in the central nervous system in rats. Neuroreport 15:67-71.

Lever IJ, Pezet S, McMahon SB, Malcangio M (2003) The signaling components of sensory fiber transmission involved in the activation of ERK MAP kinase in the mouse dorsal horn. Mol Cell Neurosci 24:259-270.

Liu RJ, Qiang M, Qiao JT (1998) Nociceptive c-fos expression in supraspinal areas in avoidance of descending suppression at the spinal relay station. Neuroscience 85:1073-1087.

McDonald AJ, Mascagni F (1996) Cortico-cortical and cortical-amygdala projections of the rat occipital cortex: a phaseolus vulgaris leucoagglutinin study in the rat. Neuroscience 71:37-54.

Monyer H, Burnashev N, Laurie DJ, Sakmann B, Seeburg PH (1994) Developmental and regional expression in the rat brain and functional properties of four NMDA receptors. Neuron 12:529-540.

Neugebauer V (2007) The amygdala: different pains, different mechanisms. Pain 127:1-2.

Neugebauer V, Li W, Bird GC, Han JS (2004) The amygdala and persistent pain. Neuroscientist 10:221-234.

Paxinos G, Watson C (1998) The rat brain in stereotaxic coordinates. New York: Academic.

Price DD (2000) Psychological and neural mechanisms of the affective dimension of pain. Science 288:1769-1772.

Puig S, Sorkin LS (1996) Formalin-evoked activity in identified primary afferent fibers: systemic lidocaine suppresses phase-2 activity. Pain 64:345-355.

Rainville P, Duncan GH, Price DD, Carrier B, Bushnell MC (1997) Pain affect encoded in human anterior cingulate but not somatosensory cortex. Science 277:968-971.

Ren WH, Guo JD, Cao H, Wang H, Wang PF, Sha H, Ji RR, Zhao ZQ, Zhang 
YQ (2006) Is endogenous D-serine in the rostral anterior cingulate cortex necessary for pain-related negative affect? J Neurochem 96:1636-1647.

Roberson ED, English JD, Adams JP, Selcher JC, Kondratick C, Sweatt JD (1999) The mitogen-activated protein kinase cascade couples PKA and $\mathrm{PKC}$ to cAMP response element binding protein phosphorylation in area CA1 of hippocampus. J Neurosci 19:4337-4348.

Shippenberg TS, Bals-Kubik R, Herz A (1993) Examination of the neurochemical substrates mediating the motivational effects of opioids: role of the mesolimbic dopamine system and D-1 vs. D-2 dopamine receptors. J Pharmcol Exp Ther 265:31-59.

Sikes RW, Vogt BA (1992) Nociceptive neurons in area 24 of rabbit cingulate cortex. J Neurophysiol 68:1720-1732.

Sindreu CB, Scheiner ZS, Storm DR (2007) Ca2 + -stimulated adenylyl cyclases regulate ERK-dependent activation of MSK1 during fear conditioning. Neuron 53:79-89.

Sweatt JD (2001) The neuronal MAP kinase cascade: a biochemical signal integration system subserving synaptic plasticity and memory. J Neurochem 76:1-10.

Tanimoto S, Nakagawa T, Yamauchi Y, Minami M, Satoh M (2003) Differential contributions of the basolateral and central nuclei of the amygdala in the negative affective component of chemical somatic and visceral pains in rats. Eur J Neurosci 18:2343-2350.

Treisman R (1996) Regulation of transcription by MAP kinase cascades. Curr Opin Cell Biol 8:205-215.

Tzschentke TM, Schmidt WJ (1998a) Discrete quinolinic acid lesions of the rat prelimbic medial prefrontal cortex affect cocaine- and MK-801-, but not morphine- and amphetamine-induced reward and psychomotor activation as measured with the place preference conditioning paradigm. Behav Brain Res 97:115-127.

Tzschentke TM, Schmidt WJ (1998b) The development of cocaine-induced behavioral sensitization is affected by discrete quinolinic acid lesions of the prelimbic medial prefrontal cortex. Brain Res 795:71-76.

Vanhoutte P, Barnier JV, Guibert B, Pagès C, Besson MJ, Hipskind RA, Caboche J (1999) Glutamate induces phosphorylation of Elk-1 and CREB, along with c-fos activation, via an extracellular signal-regulated kinasedependent pathway in brain slices. Mol Cell Biol 19:136-146.
Vogt BA (2005) Pain and emotion interactions in subregions of the cingulate gyrus. Nat Rev Neurosci 7:533-544.

Wei F, Zhuo M (2008) Activation of Erk in the anterior cingulate cortex during the induction and expression of chronic pain. Mol Pain 4:28.

Wei F, Wang GD, Kerchner GA, Kim SJ, Xu HM, Chen ZF, Zhuo M (2001) Genetic enhancement of inflammatory pain by forebrain NR2B overexpression. Nat Neurosci 4:164-169.

Wei F, Vadakkan KI, Toyoda H, Wu LJ, Zhao MG, Xu H, Shum FW, Jia YH, Zhuo M (2006) Calcium calmodulin-stimulated adenylyl cyclases contribute to activation of extracellular signal-regulated kinase in spinal dorsal horn neurons in adult rats and mice. J Neurosci 263:851-861.

Wong ST, Athos J, Figueroa XA, Pineda VV, Schaefer ML, Chavkin CC, Muglia LJ, Storm DR (1999) Calcium-stimulated adenylyl cyclase activity is critical for hippocampus-dependent long-term memory and late phase LTP. Neuron 23:787-798.

Wu LJ, Toyoda H, Zhao MG, Lee YS, Tang J, Ko SW, Jia YH, Shum FW, Zerbinatti CV, Bu G, Wei F, Xu TL, Muglia LJ, Chen ZF, Auberson YP, Kaang BK, Zhuo M (2005) Upregulation of forebrain NMDA NR2B receptors contributes to behavioral sensitization after inflammation. J Neurosci 25:11107-11116.

Xia Z, Dudek H, Miranti CK, Greenberg ME (1996) Calcium influx via the NMDA receptor induces immediate early gene transcription by a MAP kinase/ERK-dependent mechanism. J Neurosci 16:5425-5536.

Xie YF, Wang J, Huo FQ, Jia H, Tang JS (2005) Validation of a simple automated movement detection system for formalin test in rats. Acta Pharmacol Sin 26:39-45.

Yamamura H, Iwata K, Tsuboi Y, Toda K, Kitajima K, Shimizu N, Nomura H, Hibiya J, Fujita S, Sumino R (1996) Morphological and electrophysiological properties of ACCx nociceptive neurons in rats. Brain Res 735:83-92.

Zhao MG, Toyoda H, Lee YS, Wu LJ, Ko SW, Zhang XH, Jia Y, Shum F, Xu H, Li BM, Kaang BK, Zhuo M (2005) Roles of NMDA NR2B subtype receptor in prefrontal long-term potentiation and contextual fear memory. Neuron 47:859-872.

Zhuang ZY, Gerner P, Woolf CJ, Ji RR (2005) ERK is sequentially activated in neurons, microglia, and astrocytes by spinal nerve ligation and contributes to mechanical allodynia in this neuropathic pain model. Pain 114: $149-159$. 